Discovery and Analyses of Pathogenic Variants in Explanted Hearts from Primary Cardiomyopathy Patients

Oddný Brattberg Gunnarsdóttir

Thesis for the Degree of Bachelor of Science

University of Iceland

Faculty of Medicine

Department of Health Sciences

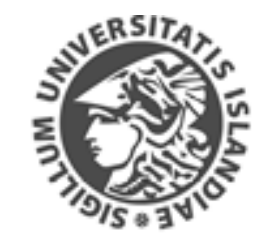

HÁSKÓLI ÍSLANDS 


\title{
Discovery and Analyses of Pathogenic Variants in Explanted Hearts from Primary Cardiomyopathy Patients
}

\author{
Oddný Brattberg Gunnarsdóttir ${ }^{1}$ \\ Supervisors: Jonathan Seidman, $\mathrm{PhD}^{2}$, and Christine Edry Seidman, $\mathrm{MD}^{2}$
}

Thesis for the Degree of Bachelor of Science

May 2019

1 Faculty of Medicine, School of Health Sciences, University of Iceland

2 Department of Genetics, Harvard Medical School, Boston, Massachusetts, USA 
Thesis for the degree of Bachelor of Science. All rights reserved. No part of this publication may be reproduced or transmitted, in any form or by any means, without written permission.

(C) Oddný Brattberg Gunnarsdóttir, 2019

Reykjavík, Iceland 2019 


\author{
Abstract \\ Discovery and Analyses of Pathogenic Variants in Explanted Hearts from Primary \\ Cardiomyopathy Patients \\ Oddný Brattberg Gunnarsdóttir ${ }^{1}$, Jonathan Seidman, $\mathrm{PhD}^{2}$, and Christine E. Seidman, $\mathrm{MD}^{2}$ \\ 1 Faculty of Medicine, School of Health Sciences, University of Iceland \\ 2 Department of Genetics, Harvard Medical School, Boston, Massachusetts, USA
}

Introduction: Dilated cardiomyopathy (DCM) and hypertrophic cardiomyopathy ( $\mathrm{HCM})$ are disorders of the myocardium that affect the structure and function of the heart. The primary aim of this study was to discover damaging genetic variants in myocardial tissue from patients with DCM or HCM who underwent heart transplantation.

Methods: Whole exome sequencing was performed on myocardial tissue from 103 explanted hearts; $80 \mathrm{DCM}, 13 \mathrm{HCM}$ and 10 donor hearts that were not implanted. Sanger sequencing was performed to confirm the loss of function variants in genes known to be linked to cardiomyopathy. RNA sequencing was conducted to confirm copy number variation deletions detected in the cohort. Burden analysis was performed by comparing the frequency of variants found in the study cohort to the frequency in the population database gnomAD.

Results: Loss of function variants, deleterious missense variants, or copy number variation deletions, collectively described as damaging variants, were identified in cardiomyopathy genes in 45 of all 103 samples (43.7\%). Damaging variants were identified in 37 of $80 \mathrm{DCM}$ samples $(46.3 \%), 5$ of $13 \mathrm{HCM}$ samples (38.4\%), and 3 of 10 control samples (30.0\%). Analyses of variants detected in control samples showed no definitive pathogenic variants. All the 28 loss of function variants in cardiomyopathy genes found in the cardiomyopathy cases were confirmed by Sanger sequencing. Two copy number variation deletions were detected and confirmed in the cohort, both in TTN. Burden analyses showed that the genes MDK, UBE2I, and MATR3, all highly expressed in the heart but not previously linked to cardiomyopathy, had a higher frequency of variants in the DCM cohort compared to the reference population with genome-wide significance $(p \leq 2.5 \mathrm{E}-06)$.

Conclusions: The frequency of damaging variants that are likely pathogenic is higher in DCM cases in this cohort compared to previous studies. This could indicate that patients who develop end-stage DCM are more likely to have a genetic cause for their disease. It is also possible that more variants are found by sequencing DNA extracted from cardiac tissue versus blood. The proportion of pathogenic variants in the HCM group is slightly lower than expected in a HCM cohort which may be explained by the low number of HCM patients in the study cohort. 


\section{Acknowledgements}

Many thanks to Jon and Kricket Seidman for giving me the opportunity to work with them and all the excellent people at their lab. Special thanks to Yuri Kim, Daniel Reichart, Jonathan Willcox, Joshua Gorham, and Steven DePalma for their guidance. Thanks to everyone at the Seidman lab for welcoming me and introducing me to research and laboratory work. I would also like to thank my parents and sisters for making this possible and Gunnar for his endless support and understanding. 


\section{Table of Contents}

Abstract

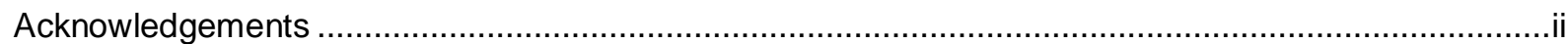

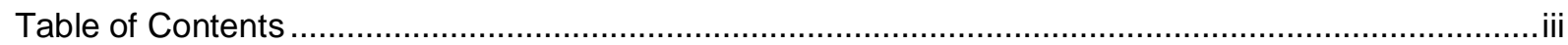

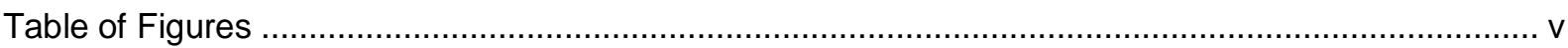

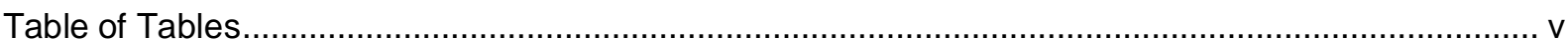

Table of Acronyms and Abbreviations …..................................................................................

$1 \quad$ Introduction

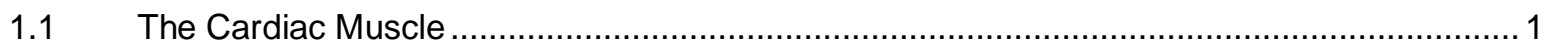

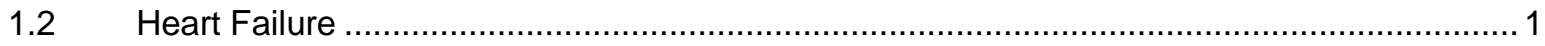

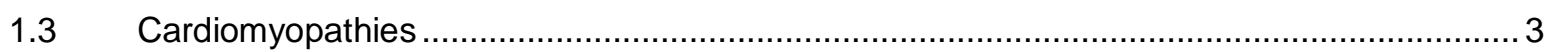

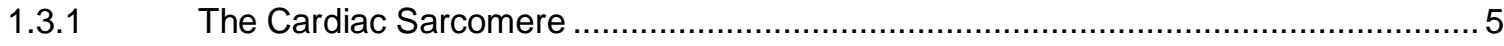

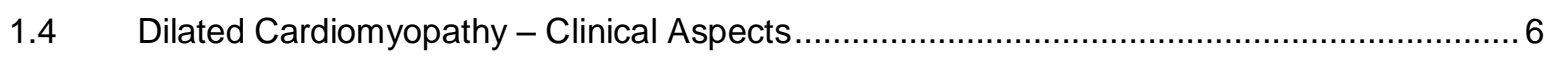

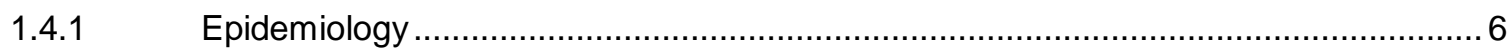

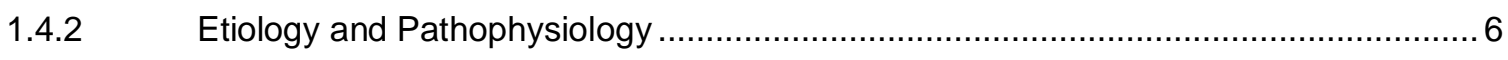

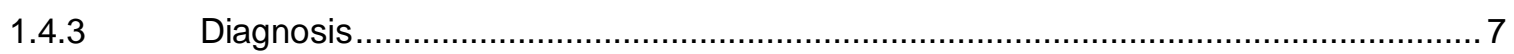

1.4.4 Symptoms, Treatment and Outcome ………...................................................

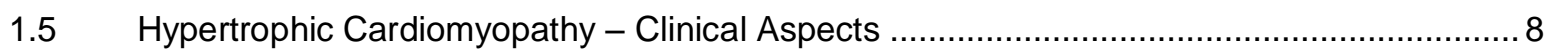

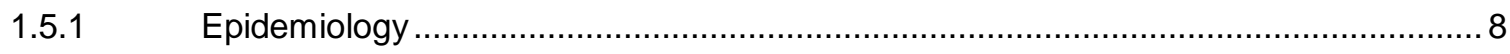

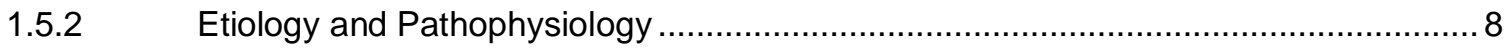

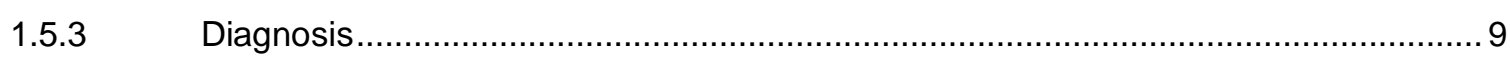

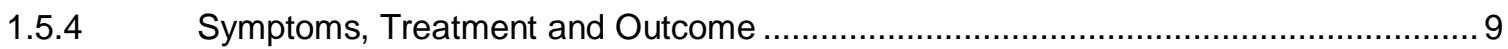

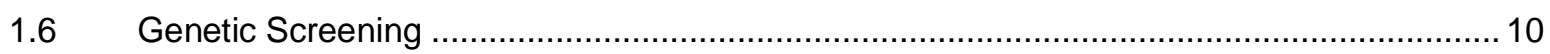

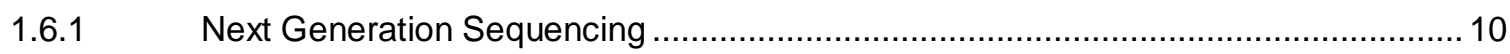

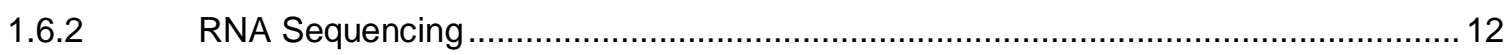

1.6.3 Genetic Diagnosis and Known DCM Genes ......................................................... 12

1.6.4 Genetic Diagnosis and Known HCM Genes ......................................................... 13

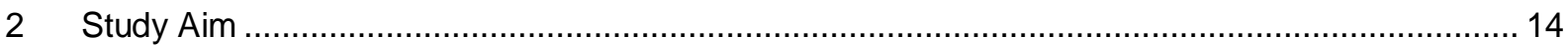

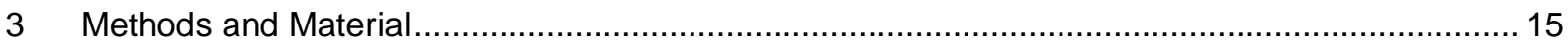

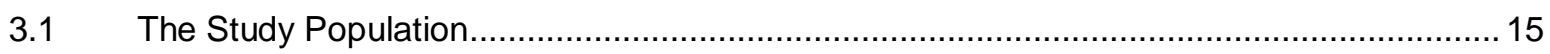

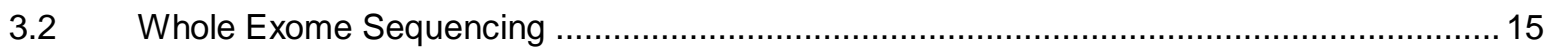

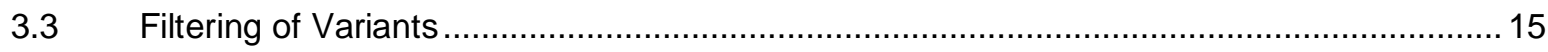

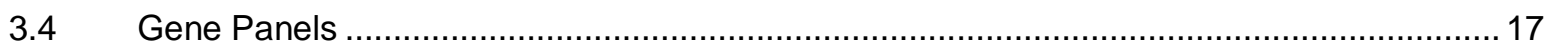

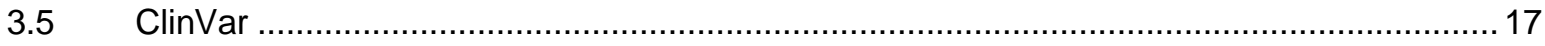

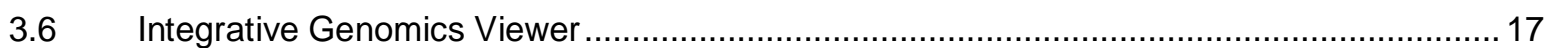




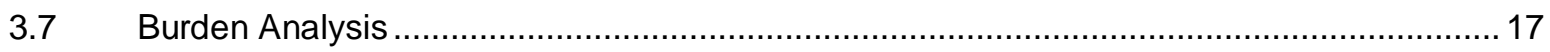

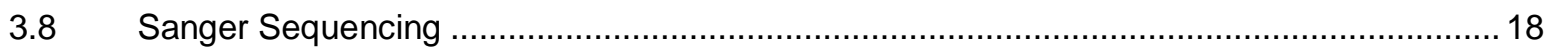

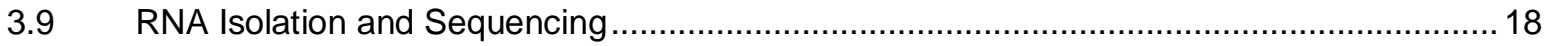

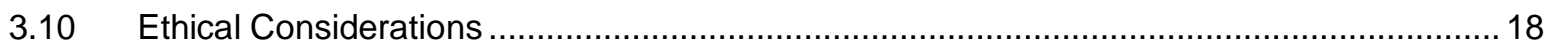

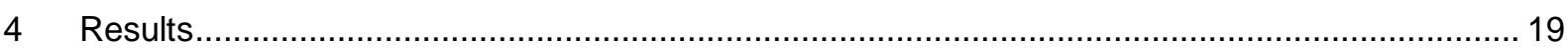

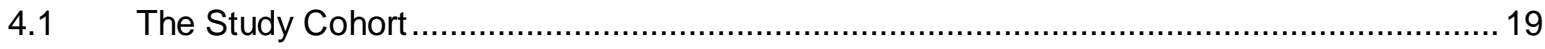

$4.2 \quad$ Identification of Rare Damaging Variants ................................................................... 19

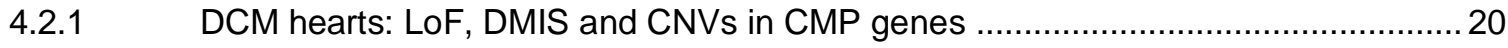

4.2.2 HCM hearts: LoF and DMIS Variants in CMP Genes .......................................... 21

4.2.3 Control Donor Hearts: LoF and DMIS Variants in CMP Genes ................................22

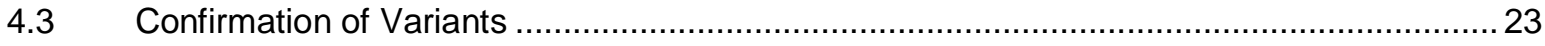

4.4 Non-CMP Genes With Enriched Frequency of Variants ….............................................. 24

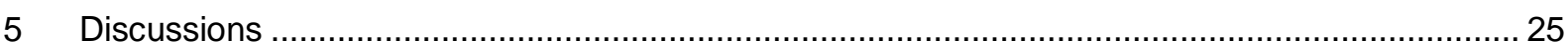

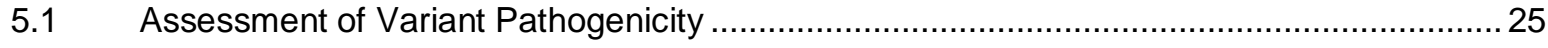

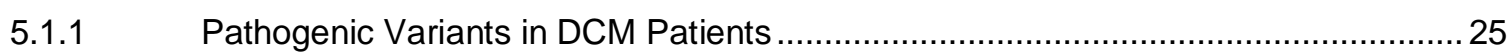

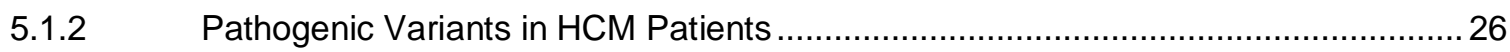

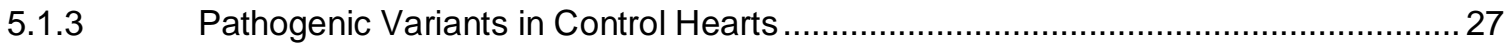

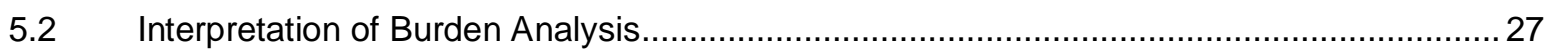

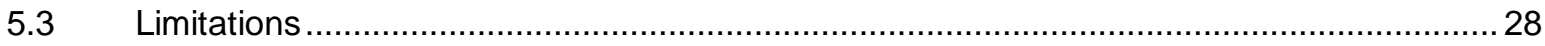

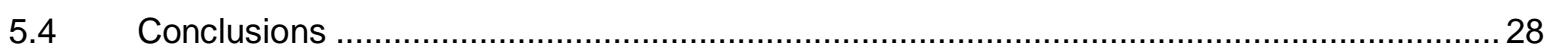

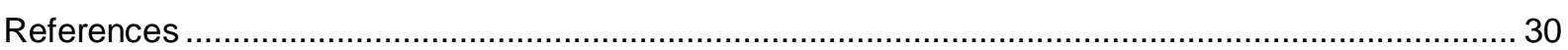




\section{Table of Figures}

Figure 1.1 The gross anatomy of a healthy heart (5) ................................................................. 1

Figure 1.2 The neurohormonal activation in heart failure (9) ..........................................................

Figure 1.3 A macroscopic view of cardiac remodeling of the left ventricle in DCM and HCM (6) ..........3

Figure 1.4 The two main classifications of cardiomyopathies.......................................................... 4

Figure 1.5 The cardiac sarcomere and the genes encoding its proteins (17) . ................................... 5

Figure 1.6 A microscopic view of healthy, HCM and DCM cardiac tissues (6) .................................. 6

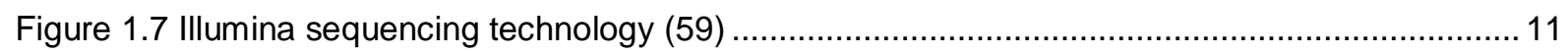

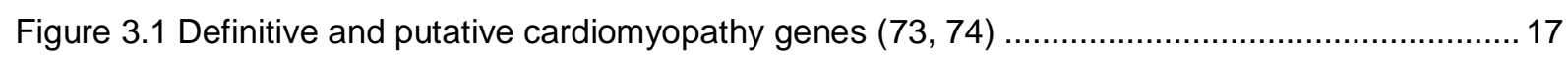

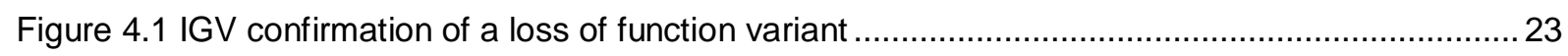

Figure 4.2 Confirmation of a loss of function variant by Sanger sequencing ..................................... 23

\section{Table of Tables}

Table 4.1 The study cohort 19

Table 4.2 Numbers of variants found in CMP genes in all tissue samples ........................................ 19

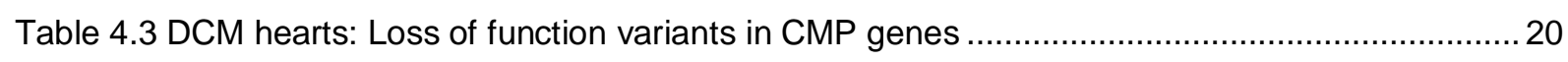

Table 4.4 DCM hearts: Deleterious missense variants in CMP genes ..............................................2 21

Table 4.5 DCM hearts: Copy number variation deletions in CMP genes .........................................2 21

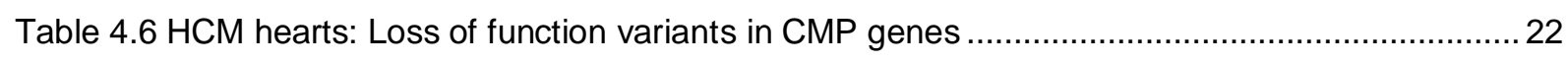

Table 4.7 Deleterious missense variants in CMP genes in HCM patients .........................................22

Table 4.8 Control donor hearts with LoF or DMIS variants in CMP genes.......................................... 22

Table 4.9 Non-CMP genes with enriched frequency of LoF variants .............................................. 24

Table 4.10 Loss of function variants in non-CMP genes with enriched frequency of variants ..............24 


\section{Table of Acronyms and Abbreviations}

\begin{tabular}{|c|c|}
\hline ACCF & American College of Cardiology Foundation \\
\hline ACE & Angiotensin-converting enzyme \\
\hline ACEI & Angiotensin-converting enzyme inhibitors \\
\hline AF & Atrial fibrillation \\
\hline AHA & The American Heart Association \\
\hline ARB & Angiotensin receptor blockers \\
\hline ARNI & Angiotensin receptor-neprilysin inhibitors \\
\hline ARVC & Arrhythmogenic right ventricular cardiomyopathy \\
\hline CMP & Cardiomyopathy \\
\hline CMR & Cardiac magnetic resonance \\
\hline CNS & Central nervous system \\
\hline CRT & Cardiac resynchronization therapy \\
\hline CRT-D & Cardiac resynchronization therapy defibrillator \\
\hline $\mathrm{DCM}$ & Dilated cardiomyopathy \\
\hline DRX & Disordered-relaxation \\
\hline EF & Ejection fraction \\
\hline ELC & Essential light chain \\
\hline ESC & The European Society of Cardiology \\
\hline $\mathrm{HCM}$ & Hypertrophic cardiomyopathy \\
\hline ICD & Implantable cardioverter defibrillator \\
\hline IHM & Interacting head motifs \\
\hline LV & Left ventricle \\
\hline LVAD & Left ventricular assist device \\
\hline LVOT & Left ventricular outflow tract \\
\hline LVW & Left ventricular wall \\
\hline MR & Mineralocorticoid receptor \\
\hline MRA & Mineralocorticoid receptor antagonist \\
\hline NGS & Next generation sequencing \\
\hline RAAS & Renin angiotensin aldosterone system \\
\hline $\mathrm{RCM}$ & Restrictive cardiomyopathy \\
\hline RLC & Regulatory light chain \\
\hline ROS & Reactive oxygen species \\
\hline SAM & Systolic anterior motion \\
\hline SCD & Sudden cardiac death \\
\hline SNS & Sympathetic nervous system \\
\hline SRX & Super-relaxation \\
\hline VCF & Variant call format \\
\hline VUS & Variants of unknown significance \\
\hline
\end{tabular}


WES

Whole exome sequencing

WGS

Whole genome sequencing 


\section{Introduction}

The increasing understanding of the human genome and the improvement of DNA sequencing over the last forty years has provided tremendous information about the underlying causes of many genetically driven diseases. Genetic testing in patients with cardiomyopathies (CMP) has revealed thousands of disease-causing variants in particular genes.(1) Meanwhile, the diagnosis of cardiomyopathies remains mainly clinically based.(2) The knowledge of the genetics of cardiomyopathies has advanced our understanding of the pathophysiology and will hopefully lead to the development of new disease specific treatment options in the manner of precision medicine.(3)

\subsection{The Cardiac Muscle}

The cardiac muscle functions like a pump which maintains the flow of blood throughout the body (Figure 1.1).(4,5) Insufficient myocardial performance results in inadequate blood supply to other organs, a condition called heart failure. Heart failure is a complex pathophysiologic complication of a variety of cardiovascular disorders, including cardiomyopathies.(6) Heart failure affects over 23 million people worldwide.(7)

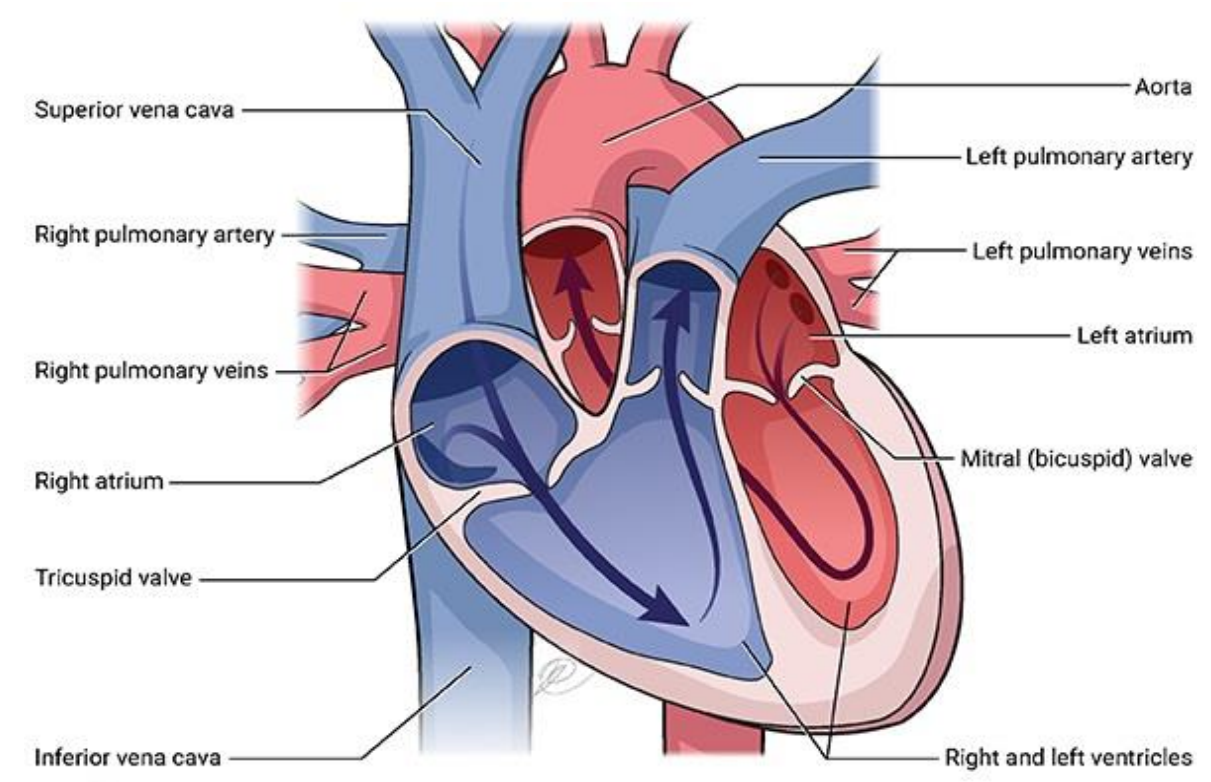

Figure 1.1 The gross anatomy of a healthy heart (5)

\subsection{Heart Failure}

The cause of heart failure is structural or functional abnormalities of the cardiac muscle that lead to increased intracardiac pressure or reduced cardiac output. Patients with heart failure can have reduced or preserved left ventricular ejection fraction $(E F) .(8)$ 
In the case of heart failure, neurohormonal activation of the sympathetic nervous system (SNS) and the renin angiotensin aldosterone system (RAAS) are the body's compensatory attempt to increase cardiac function and maintain blood pressure (Figure 1.2). In the long term, this mechanism is known to lead to the progression of heart failure and has been described as follows. Reduced EF leads to insufficient blood and oxygen supply to the rest of the body. Peripheral arterial baroreceptors in the aorta and carotid sinus sense the reduction in the pressure of blood pumping out of the left ventricle (LV) into the circulatory system. The baroreceptors initiate neurohormonal activation, which is the body's attempt to sustain cardiovascular homeostasis with a cascade of compensatory mechanisms. The central nervous system (CNS) receives signals from the baroreceptors and activates efferent pathways of the SNS, while the parasympathetic tone decreases.(9) The activation of the SNS results in the increased release and decreased uptake of norepinephrine at adrenergic nerve endings. In the short term, this leads to the augmentation of LV contractility and the heart rate, which helps sustain cardiac output. Furthermore, it increases the systemic vascular resistance and the ventricular preload by increasing systemic vasoconstriction and venous tone.(10) The juxtaglomerular apparatus senses the decrease in blood flow mediated by the vasoconstriction of the afferent renal artery. This activates the RAAS, with increased renin release into the afferent arteriole. Increased SNS signals further mediate the release of

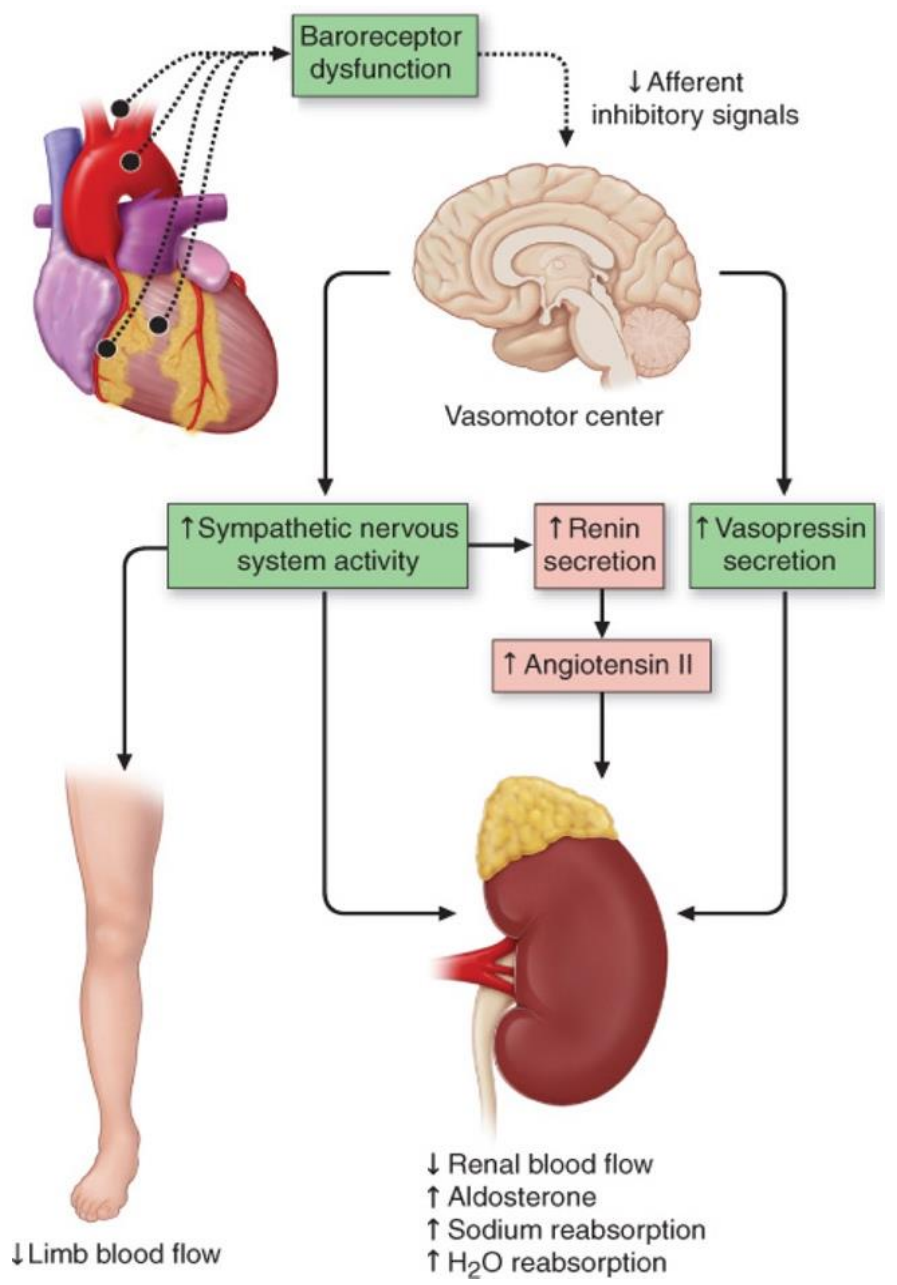

Figure 1.2 The neurohormonal activation in heart failure (9) 
renin by activating $\beta 1$-adrenergic receptors on the juxtaglomerular apparatus. Renin converts angiotensinogen to angiotensin I, which is cleaved by angiotensin-converting enzyme (ACE) into the potent vasoconstrictor angiotensin II. Angiotensin II, besides causing vasoconstriction, mediates the release of aldosterone from the adrenal glands.(9) Aldosterone has widespread effects on the cardiovascular system, causing cell death, fibrosis, hypertrophy and endothelial dysfunction. Aldosterone activation of mineralocorticoid receptors (MR) results in sodium retention and potassium excretion.(11)

The main symptoms of heart failure are dyspnea, peripheral edema, and fatigue. Dyspnea occurs due to raised left atrial pressure, which leads to lung congestion, as well as abnormalities of the functioning of chemoreceptors and respiratory muscles. Skeletal muscle hypoperfusion instigates fatigue, and fluid retention leads to peripheral edema. Palpitations due to reduced effort tolerance and cachexia due to intestinal congestion and chronic cytokine activation are symptoms of end-stage heart failure.(8) As cardiac function deteriorates, the risk increases of cardiac thromboembolic events and arrhythmias such as atrial fibrillation (AF) or ventricular arrhythmias and sudden cardiac death (SCD).(12, 13)

\subsection{Cardiomyopathies}

Cardiomyopathies are heterogenous disorders of the myocardium that affect the structure and function of the heart.(14) Two of the most common cardiomyopathies are dilated cardiomyopathy (DCM) and hypertrophic cardiomyopathy (HCM). DCM and HCM have opposing anatomical and pathological presentations in many ways (Figure 1.3), but ultimately, both lead to heart failure.(6)
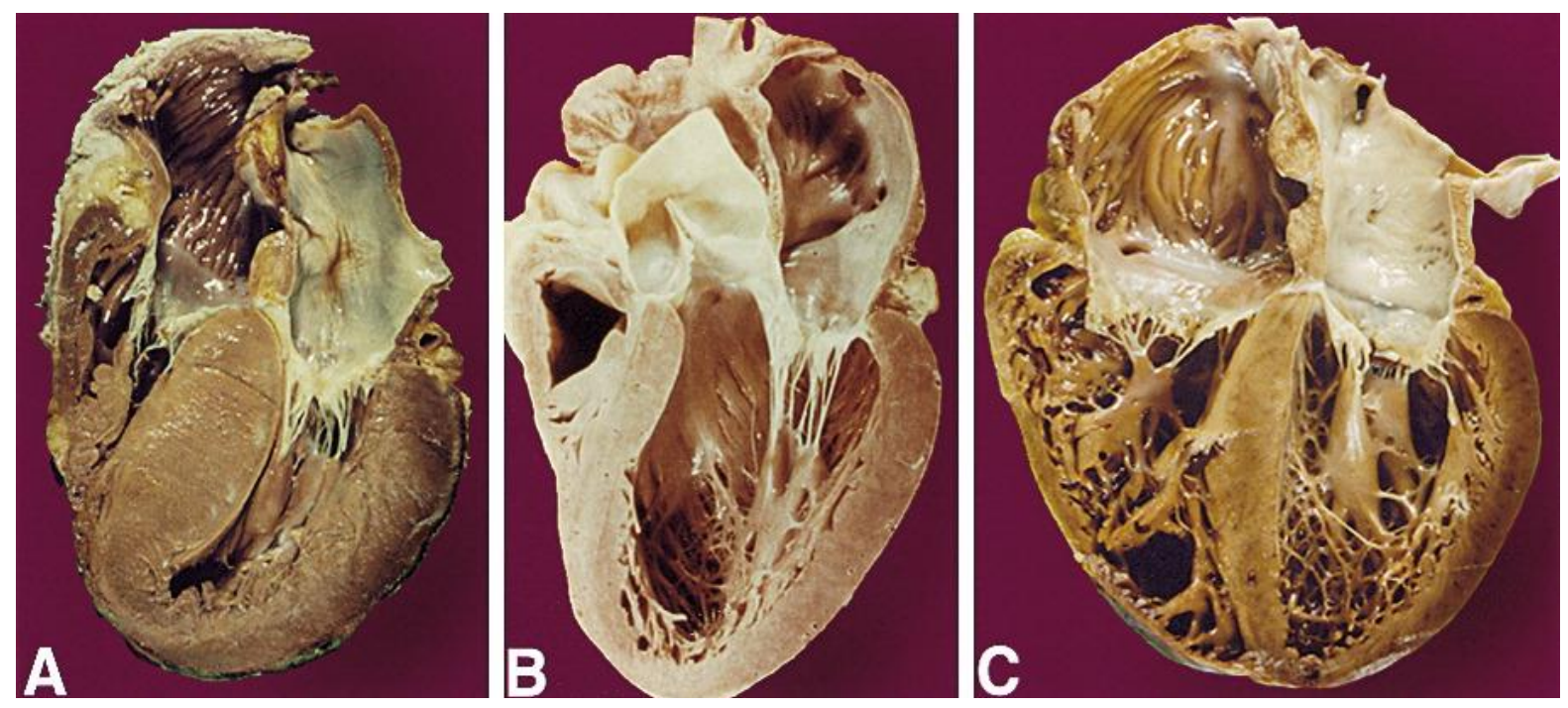

Figure 1.3 A macroscopic view of cardiac remodeling of the left ventricle in DCM and HCM (6)

Image (B) presents a healthy heart with a normal left ventricular wall thickness. Image $(A)$ is an $\mathrm{HCM}$ heart with severe thickness of the left ventricular wall, as opposed to image (C), which is a DCM heart with a dilated left ventricle and thin left venticular walls.

Through the years, diseases of the cardiac muscle have been classified in several ways. The latest classification of cardiomyopathies published by the American Heart Association (AHA) in 2006 
divides cardiomyopathies into primary and secondary cardiac diseases (Figure 1.4). Primary cardiomyopathies can be defined as diseases merely involving the myocardium in the absence of another pre-existing disease that secondarily could have this effect on the myocardium. The primary cardiomyopathies are further divided into genetic, mixed (genetic and non-genetic), and acquired cardiac diseases. Figure 1.4 (left) shows the AHA's distribution of primary diseases into genetic, mixed, or acquired cardiomyopathies.(1) The AHA defines secondary cardiomyopathies as diseases caused by a systemic condition affecting the function and structure of the myocardium. (1)

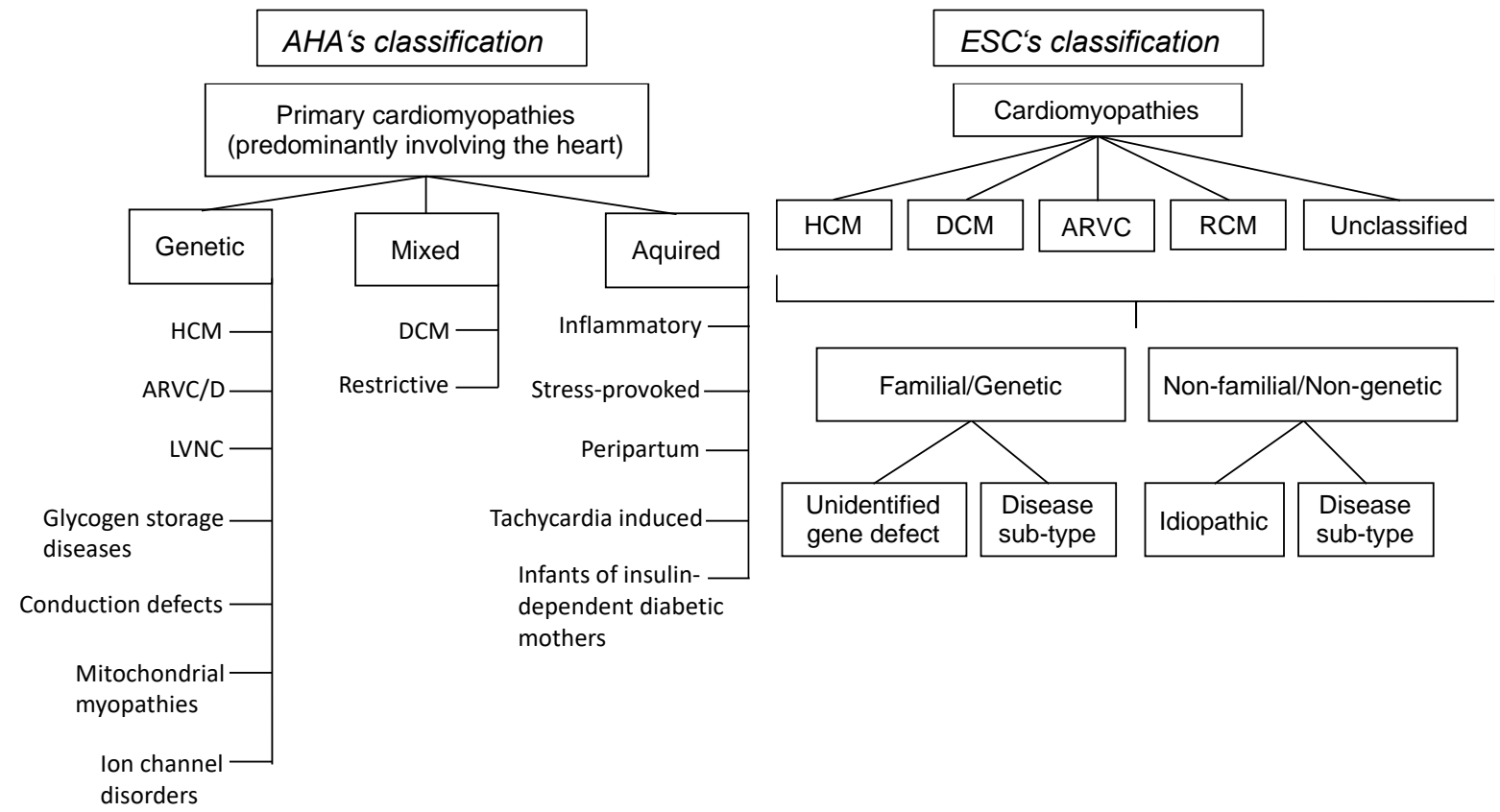

Figure 1.4 The two main classifications of cardiomyopathies $(1,15)$

AHA's classification (to the left) and ESC's classification (to the right)

The European Society of Cardiology (ESC) working group on myocardial and pericardial diseases proposed a new classification of cardiomyopathies in 2008 that is more clinically oriented in comparison to the AHA's classification. The ESC classifies cardiomyopathies into specific morphological phenotypes and then subclassifies them into familial or non-familial types (Figure 1.4, right). The main morphological phenotypes are HCM, DCM, restrictive cardiomyopathy (RCM), arrhythmogenic right ventricular cardiomyopathy (ARVC), and unclassified diseases like left ventricular non-compaction (LVNC) and stress ("Tako-Tsubo") cardiomyopathy.(15) The ESC's definition of non-familial cardiomyopathies is the presence of the disease in a patient without any other family member affected. Non-familial cardiomyopathies are further divided into idiopathic and acquired cardiomyopathies. In the case of idiopathic cardiomyopathies, no specific cause of the disease is identified. In acquired cardiomyopathies, the dysfunction of the ventricles is a complication of another disease or condition. The ESC defines cardiomyopathies as familial if the same disease or a phenotype that is thought to be caused by the same genetic variation is found in more than one family member. Most familial cardiomyopathies are monogenic disorders. The ESC classifies de novo mutations that cause cardiomyopathy as familial since the disorder can be transmitted to the patient's children.(15) 


\subsubsection{The Cardiac Sarcomere}

The cardiac sarcomere is the contractile unit of the heart muscle. HCM and DCM are often caused by variants in genes that encode components of the cardiac sarcomere.(16) The sarcomere unit is made up of thick and thin filaments which overlap each other, and in the phase of contraction the overlap becomes greater.

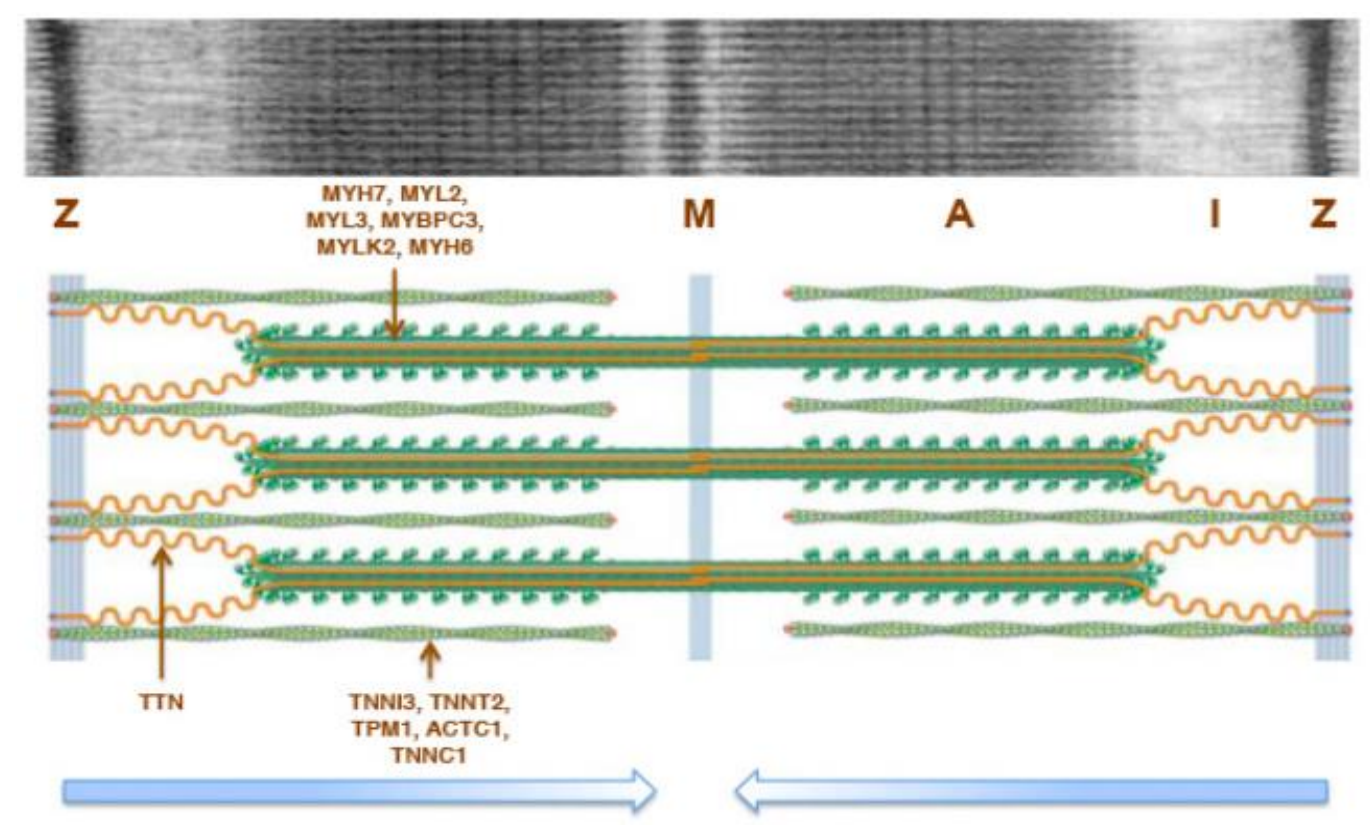

Figure 1.5 The cardiac sarcomere and the genes encoding its proteins (17) The genes MYH7, MYL2, MYL3, MYBPC3, MYLK2 and MYH6 encode proteins in the thick filaments. The genes TNNI3, TNNT2, TPM1, ACTC1 and TNNC1 encode proteins in the thin filaments. TTN encodes the protein titin. The upper part of the image shows a microscopic view of the sarcomere.

The thin filaments contain the proteins actin (encoded by the gene ACTC1), the calcium sensitive troponin complex (encoded by TNNT2, TNNI3 and TNNC1), and tropomyosin (encoded by TPM1) (Figure 1.5).(17)

The proteins of the thick filament have both motor and regulatory roles. The protein cardiac $\beta$ myosin heavy chain (encoded by MYH7) serves as a molecular motor.(17) The myosin heads (sometimes called S1) have a motor domain that hydrolyzes ATP, a regulatory domain with both essential (ELC, encoded by MYL2) and regulatory (RLC, encoded by MYL3) light chains and an S2 subfragment. Myosin tails pack together in an antiparallel manner, forming an $\alpha$-helical tail, which is the backbone of the thick filament.(18) Myosin binding protein C (cMyBP-C, encoded by MYBPC-3) is also a component of the thick filament and an essential modulator of cardiac contraction. The S2 subfragment binds CMyBP-C and titin to the backbone of the myosin.(19) Myosin heads bind to and hydrolyze ATP, which generates conformational changes in the motor domain of the myosin head, which increases the affinity for actin.(18) Phosphorylation then modulates CMyBP-C to bind actin and myosin S2, generating an interaction between actin and myosin which results in contraction.(19) 
Titin is the third major filament of the sarcomere and is the largest human protein, comprised of 35,000 amino acids.(20) The protein is divided into an elastic I-band region, an A-band region where titin binds to the thick filament, and the M-band region, where the end of the protein is embedded.(21) A spring-like function of titin produces a passive force whereby the myocytes are stretched in diastole, which defines myocardial stiffness along with the extracellular matrix.(22)

The orientation of the filaments produces bands that are visible with microscopy (Figure 1.5).(23)

\subsection{Dilated Cardiomyopathy - Clinical Aspects}

\subsubsection{Epidemiology}

DCM is a disease of the myocardium, characterized by a dilated and reduced contractile left ventricle which results in impaired systolic function.(1) The fact that DCM patients may remain asymptomatic up until notable ventricular dysfunction has taken place indicates that DCM is underdiagnosed.(24) Consequently, there is a lack of data on the true epidemiology of DCM; however, the prevalence is estimated to be $1: 250 / 500$ in adults.(25)

\subsubsection{Etiology and Pathophysiology}

DCM is considered a mixed cardiomyopathy, according to the AHA's classification, which means that it is known to have both genetic and non-genetic causes.(1) Genetic causes are thought to account for up to $35 \%$ of all DCMs. Many acquired factors are known to cause DCM, such as drugs and toxins, infectious agents, and endocrine disturbances. Myocarditis, mainly caused by viral infections, accounts for $10 \%$ of all incidents of newly diagnosed adult-onset DCM.(26)
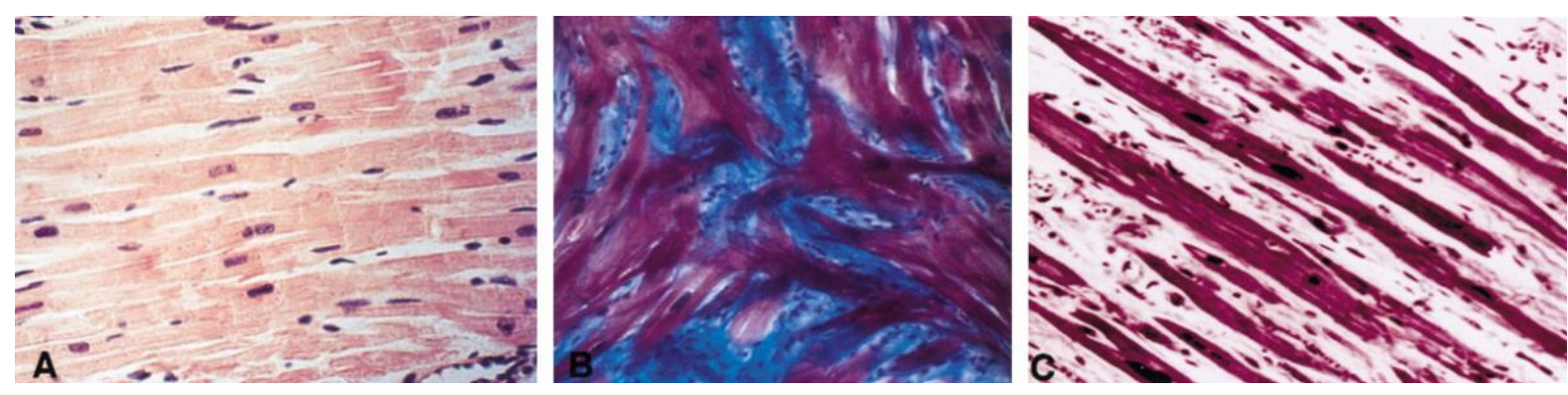

Figure 1.6 A microscopic view of healthy, HCM and DCM cardiac tissues (6)

Image (A) shows healthy cardiac tissue with a regular alignment of cardiomyocytes and no fibrosis. Image (B) shows HCM cardiac tissue with disarrayd myocytes and interstitial fibrosis (blue). Image (C) shows DCM cardiac tissue with degenerated myocytes and interstitial fibrosis (pale pink)

Pathogenic variants in specific genes can impair LV function and reduce ejection fraction (EF) by many mechanisms.(27) The neurohormonal activation that follows the reduction in EF, described in chapter 1.2, creates a vicious cycle, leading to further deterioration of LV function, irrespective of and in addition to the underlying initial cause of DCM.(9) This causes adverse remodeling of the LV by several mechanisms, including apoptosis, ischemia, fibrosis, and alterations of gene expression (Figure 1.6).(28) This results in the additional dilation of the LV, thinning of the LV walls, and the LV assuming a more spherical shape; see Figure 1.3 (C).(9) 
Additionally, an association between DCM and a higher risk of severe arrhythmia has been observed, which indicates that the cardiac conduction system is pathologically involved.(20)

\subsubsection{Diagnosis}

DCM is clinically diagnosed when the following criteria are met: 1) fractional shortening $<25 \%$ or ejection fraction $<45 \%$ and 2) left ventricular end-diastolic diameter $>117 \%$. To fulfill the criteria for idiopathic DCM, any known cause of myocardial disease must be excluded.(29) The definition of familial $D C M$ is the DCM patient has $\geq 2$ relatives with DCM that meet the clinical criteria described above or the DCM patient has a relative that died suddenly without explanation before the age of 35.(20)

An assessment of the size and function of the LV is obtained using imaging methods, most commonly, echocardiography and cardiac magnetic resonance (CMR).(20) CMR can provide additional knowledge about the amount of fibrosis in the heart muscle with the use of contrast agents, such as gadolinium. DCM patients with fibrosis have a higher incidence of all-cause mortality, ventricular tachycardia, and SCD.(30)

\subsubsection{Symptoms, Treatment and Outcome}

As mentioned above, many patients remain asymptomatic since a mild decrease in systolic functions is not necessarily symptomatic. Symptomatic DCM is characterized by symptoms and signs of heart failure caused by reduced EF and volume.(12,13)

The treatment of DCM can be divided into symptomatic treatment and treatment that affects prognoses. The standard prognostic medical therapy consists of neuroendocrine blockage or modulation of the RAAS and the SNS with $\beta$-blockers, angiotensin-converting enzyme inhibitors (ACEI) or angiotensin receptor blockers (ARB), mineralocorticoid receptor antagonists (MRA), and the latest addition of an angiotensin receptor-neprilysin inhibitor (ARNI). In the case of deteriorated or decomposed heart failure, symptomatic treatment with loop diuretics, sometimes with the addition of vasodilators such as nitroglycerine, are used to reduce volume overload and afterload.(13, 31)

Implantable cardioverter defibrillators (ICDs) have shown to reduce mortality in patients with low LF ejection fractions.(32) Cardiac resynchronization therapy (CRT) (also known as a biventricular pacemaker) is recommended for DCM patients with low EF and broad QRS intervals on ECGs. According to guidelines from the American College of Cardiology Foundation (ACCF)/AHA, patients that remain with an ejection fraction lower than $30 \%$, despite optimal medical therapy, are candidates for ICD (also known as CRT-D). CRT therapy, with or without ICD, improves survival and quality of life, reduces hospital admissions and, when added to optimal medical therapy, provokes reverse remodeling of the LV.(26) The implantation of left ventricular assist devices (LVAD) is also a standard therapeutic option in cases of severe DCM, in which optimal medical treatment has failed. LVAD is demonstrated to improve morbidity and reduce mortality rates in patients, both as a destination therapy and in patients awaiting heart transplantation.(33) Arrhythmia monitoring, such as Holter monitors, external loop recorders, and even implantable loop recorders, are essential tools to appropriately evaluate the need for devices such as pacemakers and ICDs in DCM patients.(20) 
DCM patients with intractable advanced symptomatic heart failure despite optimal medical and device therapy may be eligible for heart transplantation. The median survival after the transplantation is more than 10 years. (26)

\subsection{Hypertrophic Cardiomyopathy - Clinical Aspects}

\subsubsection{Epidemiology}

$\mathrm{HCM}$ is probably the most commonly occurring cardiomyopathy, and data from the USA suggest that it is the most common cause of SCD among the young, including trained athletes.(34)

Epidemiologic studies on HCM have demonstrated a prevalence of 1:200-500 in the general population.(35)

\subsubsection{Etiology and Pathophysiology}

$\mathrm{HCM}$ is classified by the AHA as a primary genetic cardiomyopathy.(1) It is a monogenic disease, and pathogenic variants are detected in up to $60 \%$ of probands.(17) Unlike DCM, HCM is characterized by a hypertrophied and non-dilated LV; see Figure 1.3 (A). LV hypertrophy is commonly asymmetric and heterogenous but usually involves the interventricular septum rather than posterolateral segments. $(14,36,37)$ This condition leads to impaired relaxation of the LV, leading to diastolic dysfunction with decreased left ventricular end-diastolic volume, while the systolic function often is preserved.(17, 38) About $5-10 \%$ of HCM patients progress to an end-stage with dilated LV and decreased EF, similar to DCM.(39)

Microscopically, HCM is characterized by hypertrophied cardiomyocytes with myocardial disarray instead of the usual parallel alignment of cardiomyocytes. A variable amount of interstitial fibrosis can be seen between the cells and in vessel walls (Figure 1.6).(40)

The mitral valve is often anatomically abnormal in HCM patients, presenting with excessive leaflet tissue, abnormally long chordal or mitral leaflets, prolapse of one or both mitral leaflets, or the attachment of the papillary muscle directly onto the anterior mitral leaflet.(36) Septal hypertrophy narrows the left ventricular outflow tract (LVOT), which creates a Venturi effect, dragging the mitral valve into the LVOT during systole. This conformation can lead to systolic anterior motion (SAM), which is the displacement of the anterior mitral leaflet towards the LVOT. SAM causes mitral regurgitation and LVOT obstruction, one of the symptoms of obstructive HCM. (41)

Knowledge of the genetic basis for HCM has provided mechanistic insights and indicate that pathogenic variants destabilize interacting head motifs (IHMs) in myosin heads. Destabilization can reduce the proportion of myosin in a super-relaxed (SRX) state and increases the proportion of them in a disordered-relaxed (DRX) state in which interaction with thin filaments can occur. This model could account for why functional studies of $\mathrm{HCM}$ patients show increased contractility and decreased relaxation of the cardiac muscle, which requires increased ATP consumption.(18) The main source of ATP in cardiomyocytes are mitochondria, which also produce reactive oxygen species (ROS).(42) ROS are known to cause oxidative stress; together, this contributes to fibrosis.(43) The impairment of myocardial energetics is also thought to play a pathophysiological role in the progression of myocardial 
fibrosis in HCM patients.(44) Fibrosis is related to adverse cardiac remodeling and worsening clinical status in HCM patients. (45)

\subsubsection{Diagnosis}

The clinical diagnosis of HCM is similar to DCM, mainly made with the help of echocardiography or CMR to identify a thickened left ventricular wall.(46) A left ventricular wall (LVW) thickness of more than $15 \mathrm{~mm}$ in one or more segment of the LVW, measured by echocardiography, MRI, or computed tomography, is defined as $\mathrm{HCM}$ as long as the thickening cannot be solely explained by loading conditions. (46) On average, the thickness of the LVW in diagnosed HCM patients is $21 \mathrm{~mm}$. In extreme cases, the thickness of the LVW can reach up to $50 \mathrm{~mm}$. When the thickness is $13-14 \mathrm{~mm}$, a differential diagnosis is often required to exclude hypertension, physiologic athlete's heart, or other conditions that might explain the thickness of the septum.(35)

One-third of HCM patients have resting SAM, which causes LVOT obstruction and mitral regurgitation.(46) LVOT obstruction has an important effect on the assessment of SCD and the management of symptoms. Therefore, both $2 \mathrm{D}$ and Doppler echocardiography is recommended for all HCM patients during the Valsalva maneuver in sitting, standing, and semi-supine positions. Some patients only have dynamic LVOT obstruction provoked by exercise. Thus, patients that are not identified with LVOT obstruction in a relaxed state should be examined during exercise or with the use of glyceryl trinitrate.(36)

\subsubsection{Symptoms, Treatment and Outcome}

The main symptoms and complications of HCM are heart failure, chest pain, arrhythmias, systemic embolic events, and SCD. Regardless of this, most people with HCM live long lives without symptoms that affect their lifestyle, and many are thought to remain undiagnosed.(34)

HCM patients present with symptoms of heart failure, most commonly dyspnea and chest pain. Chest pain likely appears due to myocardial hypoperfusion and the consequential increased oxygen demand. Syncope is a serious symptom of HCM, as it has shown to be an omen of SCD.(47) Atrial fibrillation is present in about $20 \%$ of HCM patients, and evidence indicates a high association between $\mathrm{AF}$ and unfavorable disease outcomes.(48)

Symptoms and outcomes of the disease have been associated with the level of obstruction present in each case.(49) About one-third of HCM patients have LVOT obstruction at rest. Another third has LVOT obstruction provoked by exercise, and the last third has no LVOT obstruction.(50) A surgical myectomy or percutaneous septal ablation in patients with obstructive HCM results in the relief of symptoms and provides long-term survival comparable to that observed in the general population: This fact presents some of the evidence of the effect of obstruction on the symptoms of HCM.(51)

The treatment of HCM can be conceptualized into three categories: SCD risk stratification, symptomatic treatment, and stroke prevention.

All HCM patients should undergo comprehensive SCD risk stratification according to practice guidelines reported by the ACCF/AHA in 2011 and the ESC in 2014. The main risk factors for SCD are a familial history of SCD, unexplained syncope, massive LVH, non-sustained ventricular tachycardia on Holter, and hypotension with exercise. The ACCF/AHA guidelines recommend using the presence of $1-$ 
2 or more risk factors as an indication for ICD implantation. The ESC guidelines recommend using a specific risk calculator. $(46,52)$

For obstructive and non-obstructive HCM patients with symptoms of chest pain and dyspnea, B-blockers or verapamil is recommended as a treatment. Disopyramide can be combined with Bblockers or verapamil in patients with obstructive HCM. Oral diuretics may be added in obstructive and non-obstructive HCM with persistent dyspnea or congestion. Septal reduction therapy, such as septal myectomy or alcohol septal ablation, is indicated for patients who have severe symptoms that affect everyday life, despite medical therapy. Non-obstructive HCM patients who develop systolic dysfunction with the ejection fraction $\leq 50 \%$ should be treated according to guidelines for patients with heart failure of similar severity for other reasons.(52) The use of LVAD is less common in end-stage HCM patients compared to DCM patients, since the reduced LV cavity in HCM patients complicates the implantation of LVAD.(53) Non-obstructive HCM patients with end-stage heart failure not responding to other optimal treatment can be considered for heart transplantation. For asymptomatic HCM patients, neither a pharmaceutical nor septal reduction therapy is recommended.(52)

For stroke prevention, lifelong therapy with anticoagulants is recommended for HCM patients with paroxysmal, persistent, or permanent AF.(46)

\subsection{Genetic Screening}

Watson and Crick described the structure of deoxyribonucleic acid in 1953.(54) This seminal publication greatly increased the understanding of how nucleic acids are aligned to encode proteins. The order of nucleic acids in DNA determines the heredity of organisms and most biochemical properties of life. Hence, it is essential to be able to sequence DNA to connect genotype with phenotype.(55)

Sanger sequencing is the founding method of DNA sequencing. It came as a breakthrough in 1977 when the Sanger dideoxy technique, or "chain-termination," was established. Sanger sequencing and other first-generation sequencing methods alone can only read sequences less than $1 \mathrm{~kb}$ in length. Techniques such as polymerase chain reaction (PCR) further improved Sanger's technique, enabling it to analyze longer fragments of DNA.(55) Sanger sequencing is precise and useful when high throughput is not required; however, it is time consuming and expensive to analyze large numbers of genes.(56)

\subsubsection{Next Generation Sequencing}

Next-generation sequencing (NGS) is a newer and still-developing method of DNA sequencing technology that has enabled high-throughput sequencing. This has made the analysis of mutations in the genome cheaper and faster.(56)

NGS technologies are further divided into second generation, third generation, and so on, since the methodology is still evolving. Second-generation sequencing is divided into category sequencing by hybridization and sequencing by synthesis, the latter including the further development of Sanger sequencing.(57) Sequencing by hybridization is a method where specific DNA sequences are attached to a surface in a 2-D array. A mixture of nucleic acids which have been labeled with chemicals is then spread over the surface. Hybridization occurs between identical nucleic acids in the mix and on the surface, which binds the nucleic acids in the mix to the surface. This method is used for measuring concentrations of nucleic acids in a solution.(58) The most widely used second-generation sequencing 
technology, accounting for over $70 \%$ of the NGS market, is Illumina sequencing.(59) It is based on a "bridge amplification" technique. Oligonucleotide sequences are placed on a glass slide. About 500 base pair long DNA molecules, with adaptors complementary to oligonucleotide sequences attached to each end, are used as substrates for the amplification of synthesis reactions that occur on the glass slide. DNA then creates clonal clusters, consisting of about 1,000 copies of each oligonucleotide sequence (Figure 1.7). This technique can be used to sequence whole genomes and exomes.(57)

NGS technologies such as Illumina are great methods in the search for causative mutations in patients and relatives of patients with hereditary cardiovascular conditions since they offer high throughput sequencing. Furthermore, some of the genes known to be responsible for cardiovascular diseases are too large to be analyzed by Sanger sequencing alone. Whole genome sequencing (WGS) covers nearly all the sequences in the genome (both exons and introns), and whole exome sequencing (WES) covers all exons (protein coding sequences) in the genome.(60) A downside of this elegant technology is that, when analyzing large numbers of genes, variants of unknown clinical significance (VUS) are likely to be identified. This makes it challenging for physicians and clinical geneticists to interpret the clinical relevance of NGS results.(56)
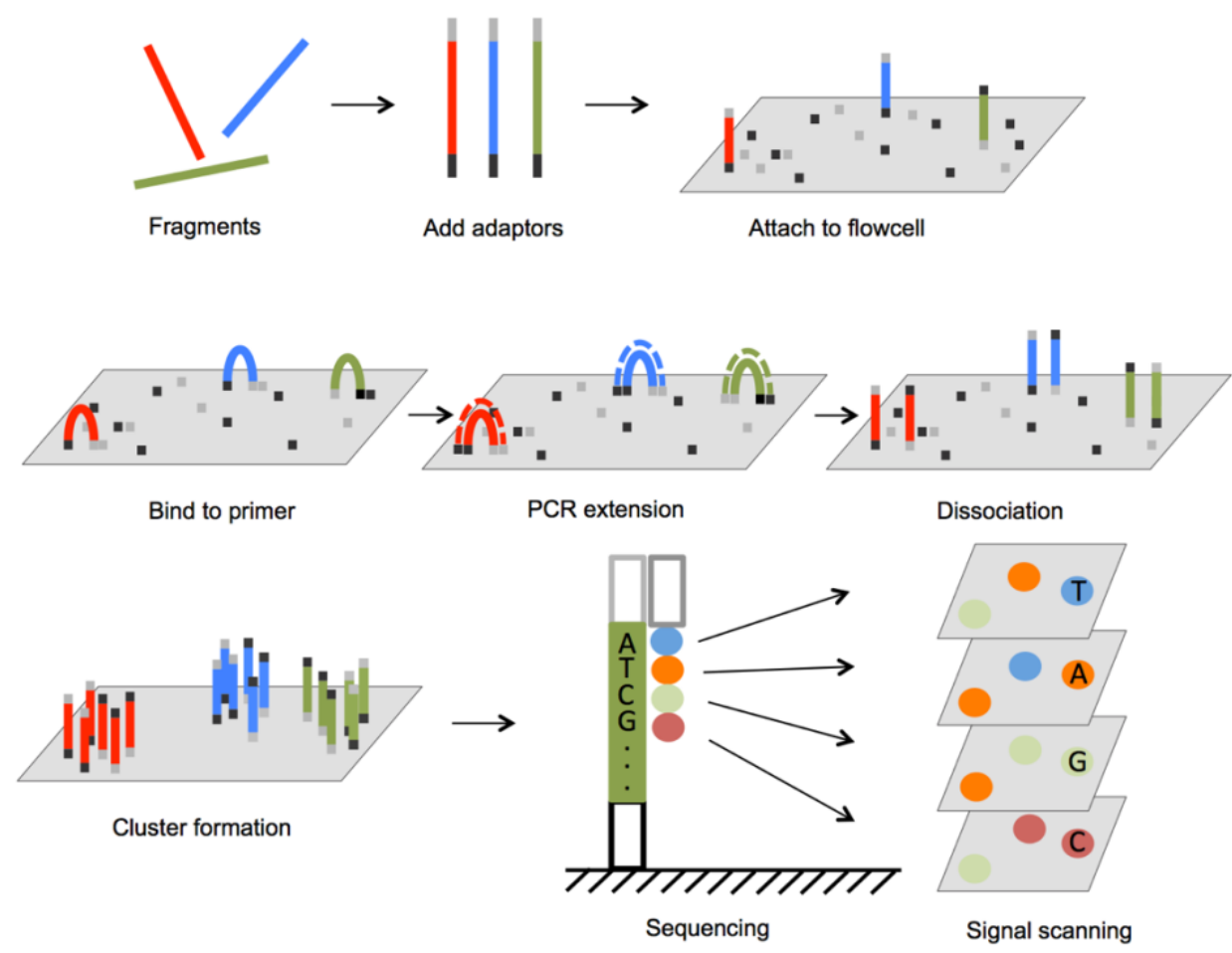

Figure 1.7 Illumina sequencing technology (59)

NGS can be performed on targeted gene panels, which are sets of genes known to be associated with specific diseases. Results of using the targeted gene panel method have shown to be of similar quality as Sanger sequencing. Too-restrictive targeted gene panels offer the risk of missing potential disease-causing variants, which could hinder a genetic diagnosis. Conversely, the analysis of WGS and WES can be time-consuming, and many VUS and irrelevant findings that burden the diagnostic process are likely to be found.(60) 
Genetic testing in general does not alter treatments or outcomes in the individual cardiomyopathy patient, with the exception of metabolic cardiomyopathies, such as Fabry or Pompe disease.(61) The finding of a disease-causing variant in an affected patient can be used for the cascade screening of relatives after careful genetic counseling.(3) Relatives found to be carriers of diseasecausing variants would be offered clinical follow-up, and non-carriers would be discharged from followup.(61)

\subsubsection{RNA Sequencing}

The transcriptome of a cell is a collection of RNA molecules that have been transcribed from genes. RNA sequencing (RNA-seq) is a technique that gives insight into the transcriptome of cells. The data provided by RNA-seq enable the interpretation of functional elements of the genome in cells by revealing gene expression, alternative splicing, and allele-specific expression. RNA-seq is performed by extracting and isolating RNA from tissue and then converting the RNA to complementary DNA (cDNA). Next, cDNA is sequenced on an NGS platform.(62)

\subsubsection{Genetic Diagnosis and Known DCM Genes}

Pathogenic variants are thought to account for about $35 \%$ of all DCM cases. The most common transmission pattern of familial DCM is autosomal dominant inheritance. A familial pattern can be seen even though no pathogenic variant is detected. (63)

Variants that are DCM pathogenic or likely pathogenic have been reported in over 50 genes.

Of these, genes that encode components of the cardiac sarcomere are a common genetic cause for both DCM and HCM. $(26,39)$

Truncating variants in TTN, the gene encoding titin, are the most common genetic cause for DCM, accounting for $15-20 \%$ of all DCM cases.(14) After TTN, the sarcomere genes MYH7, TNNT2, and TPM1 are most frequently mutated in DCM patients, accounting for $2-4 \%$ of DCM cases.(20)

Pathogenic variants in multiple other genes that encode for components other than the sarcomere, like the nuclear membrane, cytoskeleton, outer-cellular membrane, extracellular matrix, ion channels, mitochondrial proteins, and splice-regulating proteins, have also been linked to DCM. This diversity of genes implies that the mechanisms that cause DCM are very complex and variable. Among these, pathogenic variants are most often identified in TTN and LMNA.(26)

What DCM patients should be offered genetic testing? The AHA presented a scientific statement on DCM in 2016. Their strong consensus recommended that mutation-specific genetic testing should be performed on family members and appropriate relatives of DCM patients with identified DCMcausative mutations. With a moderate consensus, they recommended genetic testing in conjunction with genetic counseling for patients with familial (Level of evidence A) and nonfamilial idiopathic cardiomyopathy (Level of evidence B).(63) However, a position statement from the ESC in 2016 recommends that genetic testing should be performed in all familial DCM cases as well as nonfamilial cases with clinical clues of the disease being genetic.(64) 


\subsubsection{Genetic Diagnosis and Known HCM Genes}

$\mathrm{HCM}$ is inherited via an autosomal dominant pattern. Variants in at least 11 genes are known to cause $\mathrm{HCM}$, and over 2,000 sarcomere variants have been identified. Many of these variants are restricted to single families, and many of them have unknown pathogenicity.(35) The variants causing $\mathrm{HCM}$ are missense mutations that substitute one amino acid for another. Missense proteins can be incorporated into sarcomeres and produce hypercontractility and poor relaxation.(17)

Disease-causing mutations in genes encoding for sarcomere proteins are found in up to $60 \%$ of HCM probands that do have a family history of $\mathrm{HCM}$ and in $40 \%$ of sporadic cases.(39) The two most common genes involved in the pathogenesis are the sarcomere genes $M Y H 7$ (beta-myosin heavy chain) and MYBPC3 (myosin-binding protein C), accounting for $75 \%$ of identified mutations. Mutations in the sarcomere protein-encoding genes MYL2, MYL3, TPM1, TNNT2, TNNI3, and ACTC1 are found in $<10 \%$ of identified mutations.(17) Pathogenic variants in the genes GLA, LAMP2, and PRKAG2 result in metabolic or infiltrative diseases that cause LV hypertrophy and mimic HCM. Life-changing therapies do exist for these diseases (Fabry disease caused by GLA variants, Danon disease caused by LAMP2 variants, and conduction diseases caused by PRKAG2 variants). Hence, it is significantly important to distinguish between these diseases and HCM.(61)

The relationship between genotype and phenotype is not clear enough to predict the prognosis of the disease. Patients with the same variant in the same gene, even in the same family, often present with diverse severities of the disease.(35) However, a few broad correlations have been seen on a gene level. Pathogenic variants in TNNT2 have been correlated with minor hypertrophy, but a larger risk of arrhythmia. Pathogenic variants in MYH7 appear to cause appreciable LVH by the second decade of life and are linked to a higher risk of heart failure and SCD. Nonetheless, pathogenic variants in MYBPC3 seem to be more associated with adult-onset LVH, even though they have also been seen in childhoodonset LVH.(39) Interestingly, missense variants are seen more frequently among pathogenic variants in $M Y B P C 3$ in children, while loss of function (LoF) variants are of higher prevalence among MYBPC3 variants seen in adult-onset disease.(65) This could indicate that missense variants in MYBPC3 are more pathogenic than LoF variants. Despite the documented correlations, decisions on treatment and other manageable factors are still only based on clinical criteria.(35)

What HCM patients should be offered genetic testing? Guidelines from the ACCF/AHA and ESC recommend that all HCM patients and their first-degree relatives undergo genetic screening and counseling. $(46,52)$ 


\section{Study Aim}

The aim of this study was to discover and analyze pathogenic genetic variants in DNA extracted from explanted hearts from end-stage DCM or HCM patients that underwent heart transplantation. 


\section{Methods and Material}

\subsection{The Study Population}

De-identified, coded cardiac tissues (see Ethical Considerations below) were received from Dr. Gavin Oudit at the University of Alberta in Canada. Adult patients with a clinical diagnosis of idiopathic DCM or HCM were eligible to participate, irrespective of gender, race, or ethnicity. The tissues were collected from 103 explanted hearts. Among these 103 samples, 80 were from patients with a clinical diagnosis of DCM, and 13 were from patients with an HCM diagnosis. Ten control samples were also obtained from healthy donor hearts that were not used for transplantation for unknown reasons.

\subsection{Whole Exome Sequencing}

DNA was studied using WES. DNA was extracted from each tissue sample using a PerkinElmer chemagic 360 instrument (Kit Cat\#CMG-1207). Sequencing libraries were prepared with a KAPA Hyper kit (Cat\# KK8504). Exome enrichment was performed with IDT xGen Human Exome Research Panel Version 1.0 (https://www.idtdna.com) and sequenced at the Yale Center for Genome Analysis on an Illumina NovaSeq instrument, as 101-base paired-end reads. Reads were mapped to the human reference genome hg19/GRCh37 using the BWA-mem aligner (66) and processed using GATK (Version 3.8) Best Practices workflows $(67,68)$. Steps included duplication marking, indel realignment, and basequality recalibration. The median on-target mean depth of coverage was $48.4 x$ (range $37.3 x$ to $131.7 x$ ). All samples had at least $85 \%$ of their target bases covered to at least $20 \mathrm{x}$, as calculated with the Picard CollectHsMetrics tool (http://broadinstitute.github.io/picard/). Variant calls were made with GATK HaplotypeCaller and GenotypeGVCFs in gvcf/multisample mode. Variant filtering was performed with GATK VQSR (variant quality score recalibration), using a truth sensitivity cutoff of 99.5 .

The raw variant call format (VCF) file was annotated with SnpEff Version 4.3t (GRCh37.75 database), dbNSFP (69), gnomAD r2.1 (70), ClinVar (December 2018 release), and the Human Genome Mutation Database (2018 Q2 release).

\subsection{Filtering of Variants}

The software program $R$, with the integrated development environment Rstudio, was used for filtering and statistically analyzing the data. The VCF file was converted to a VCF table, which is a superior format for working in R. A quality control (QC) filtration was performed on the VCF table in R, so only rare damaging variants with good quality remained. Following are the criteria for QC filtration:

- A mapping quality (MQ) score estimates the overall mapping quality of reads supporting a variant call. The criteria for the filtration was: $M Q>6$.

- The depth of reads (DP) is the number of reads of each allele that passed the variant caller's filter. The criteria for the filtration was: $D P \geq 8$.

- Altered allele balance (AAB) is the ratio of reads that support the alternative allele. Allele depth (AD) is the number of reads of each allele, including those that did not pass the variant caller's filter. The criteria for the filtration was: $A A B \geq 0.2$ if alt. $A D \geq 10, A A B \geq 0.28$ if alt.AD $<10$. 
- Genotype quality (GQ) represents the quality of the assigned genotype. The criteria for the filtration was: $\mathrm{GQ} \geq 20$.

- Allele count (AC) represents the number of alleles called in a sample. The criteria for the filtration was: $\mathrm{AC}<6$.

- The criteria for minor allele frequency in the filtration was $<1.0 \mathrm{E}-04$ in order to obtain only rare variants.

- Variants in duplicated regions were filtered out.

The types of variants sought after QC filtration were loss of function (LoF) variants, deleterious missense variants (DMIS), and copy number variations (CNV). CNVs were only searched for in the CMP genes seen in Figure 3.1. LoF and DMIS variants were searched for in all genes. Following are the filtration methods for each type of variant:

- Loss of function:

- Variants with high impact.

- Types of LoF variants: frameshift, splicing, nonsense and variants affecting start and stop codons.

- Further filtration was performed for LoF variants in TTN:

- The criteria for "Percent-sliced-in" (PSI) score was: PSI > 0.9

- Variants in the Novex-3 isoform of TTN were filtered out.

- Deleterious missense:

- Variants with moderate impact

- Variants with a MetaSVM score indicating that the missense mutation was deleterious.

- Copy number variation calling:

- CNVs were called using the statistical and computational tool XHMM.(71) Starting with bam files, GATK DepthOfCoverage was used to calculate mean per-base coverage (counting unique fragments based on reads mapping with a quality $>20$ ) across all exome targets.

- Three different exome capturing reagents were used. Accordingly, a bed file was created containing intersecting regions for the three reagents

- The resulting read depth matrix was scaled by mean-centering targets, after which principal component analysis (PCA) of the full matrix was performed. The parameters described in Ruderfer et al.'s paper in Nature Genetics 2016 for the EXAC data were used to facilitate data comparison,(72) as well as default XHMM parameters.

- All cases had CNVs called using the same parameters and in a single run. 


\subsection{Gene Panels}

Figure 3.1 shows definitive and putative cardiomyopathy genes (called CMP genes) that were interrogated in exome sequences in this thesis.(73) The genes highlighted in grey are definitive DCM genes, and the ones typed in bold are definitive HCM genes. (74) Other genes in the panel are putative CMP genes, which means that their connection to the pathogenesis of the diseases is not as definite.

\begin{tabular}{lllllllllll} 
ABCC9 & APOA1 & DES & EMD & FOXD4 & ILK & MYH6 & PDLIM3 & SCN5A & SOD2 & TNNC1 \\
ACTC1 & BAG3 & DMD & EYA4 & FXN & LAMA2 & MYH7 & PLEC & SDHA & SYNE1 & TNNI3 \\
ACTN2 & CAV3 & DNAJC19 & FHL2 & HADHA & LAMA4 & NEB & PLN & SGCA & SYNM & TNNT2 \\
ALMS1 & CHRM2 & DSG2 & FKRP & HFE & LAMP2 & NEBL & PRKCDBP & SGCB & TAZ & TPM1 \\
ALPK3 & CRYAB & DSP & FKTN & HOPX & LDB3 & NEXN & PSEN1 & SGCD & TBX2O & TTN \\
ANKRD1 & CSRP3 & DTNA & FLNC & HSPB7 & LMNA & NFKB1 & PSEN2 & SGCG & TCAP & VCL \\
& CTF1 & & FLT1 & \multicolumn{3}{c}{ MYBPC3 } & RBM2O & & TMPO &
\end{tabular}

Figure 3.1 Definitive and putative cardiomyopathy genes $(73,74)$

Highlighted genes are definitive dilated cardiomyopathy genes, bolded genes are definitive hypertrophic cardiomyopathy genes.

In addition to the CMP genes, I studied a panel of 4,420 genes with high heart expression (HHE), to determine if damaging variants in these genes might cause cardiomyopathy.(75)

\subsection{ClinVar}

ClinVar is a collection of reports regarding the relationship between variations in the human genome and phenotype. To discover if variants identified in this study were previously reported in human patients, the variants were sought in ClinVar. If a rare pathogenic variant found in the study cohort had been annotated as pathogenic or likely pathogenic in ClinVar, the likelihood of the variant being pathogenic is increased.

\subsection{Integrative Genomics Viewer}

The integrative genomics viewer (IGV) is a high-performance visualization tool used for the interactive exploration of integrated genomic datasets.(76) IGV was used to confirm LoF and DMIS variants in CMP genes and LoF variants in HHE genes. It was also used to interpret RNA sequencing results by searching for heterozygous SNPs in the regions of detected CNV deletions, since heterozygous SNPs should not be present in a homozygous region.

\subsection{Burden Analysis}

A binominal test is a simple statistical test that is used to compare two independent binomial proportions.(77) It was performed to compare frequencies of pathogenic variants found in genes in the cohort to their frequency in the same genes in the Genome Aggregation Database (gnomAD). While pathogenic variants in genes in the CMP panel can mostly be interpreted as disease-causing, the significance of the pathogenicity of variants in genes that are not in the CMP gene panel was estimated by burden analyses. GnomAD consists of data from WES and WGS from 141,456 unrelated individuals. The data span 125,748 exomes and 15,708 genomes. Individuals that have experienced severe 
pediatric disease and their first-degree relatives have been removed from the dataset so the frequency of severe disease should be equivalent to or lower than in the general population. The data are a collection from a variety of large-scale sequencing projects. The gnomAD database is available on the web (www.gnomad.broadinstiture.org).

When a multi-testing correction for 20,000 genes is considered, a p-value lower than 2.5e-06 was estimated for the findings to be significant across the genome.

\subsection{Sanger Sequencing}

To further confirm the LoF variants identified in known CMP genes, Sanger sequencing was performed for the relevant amplicons. The software Primer3 Version 0.4.0 was used to design sequencing primers.(78) The product size was 150-250 base pairs. The DNA was amplified by polymerase chain reaction (PCR), which was performed using a Surecycler 8800 Thermal cycler from Agilent Technologies (Agilent Technologies, California, USA). Thermal cycling was performed with the beginning temperature of $98^{\circ} \mathrm{C}$ for 30 seconds, followed by 30 cycles $\left(10\right.$ seconds at $98^{\circ} \mathrm{C} ; 30$ seconds at $60^{\circ} \mathrm{C} ; 30$ seconds at $72^{\circ} \mathrm{C}$ ) and then put on hold at $4^{\circ} \mathrm{C}$. The quality of the product was assessed using a TapeStation 2200 (Agilent Technologies, California, USA). Amplicons were sequenced by Genewiz, New Jersey, USA.

\subsection{RNA Isolation and Sequencing}

RNA-seq was performed on samples with detected CNV deletions to examine the RNA expression in the region of the detected deletion. RNA was extracted from excised tissues using TRIzol (Life Technologies, Waltham, Massachusetts, USA). The quality and quantity of the product was assessed using a TapeStation 2200 (Agilent Technologies, California, USA). Two rounds of polyA selection were done before the synthesis of cDNA. Moreover, cDNA libraries were constructed from human reference genome hg19, and RNA-seq libraries were constructed using the Nextera XT DNA Library Preparation Kit (Illumina). Libraries were sequenced on the Illumina platform (NextSeq500) before they were aligned to the human reference hg19 using STAR aligner software. The total number of reads of each transcript was normalized to one million.

\subsection{Ethical Considerations}

This study was performed using protocols that were reviewed and approved by the human research committees at the University of Edmonton (Canada) and Brigham and Women's Hospital at Harvard Medical School (USA). Adult patients with a clinical diagnosis of idiopathic DCM or HCM were eligible to participate, irrespective of gender, race or ethnicity. All subjects provided written informed consent. LV cardiomyopathy tissues were obtained at the time of cardiac transplantation or autopsy that were performed solely for clinical purposes. Tissues were labelled with a de-identified code and transferred to the research laboratory for DNA extraction and sequencing. Similarly, clinical information was provided to the research laboratory using de-identified codes. 


\section{Results}

\subsection{The Study Cohort}

Table 4.1 shows the distribution of the samples into DCM, HCM, and controls, with additional information about gender, age at explantation (age of death for patients with healthy hearts), and whether the patients had LVAD before the transplantation.

Table 4.1 The study cohort

\begin{tabular}{llll}
\hline & $\mathrm{DCM}(\mathrm{n}=80)$ & $\mathrm{HCM}(\mathrm{n}=13)$ & Control $(\mathrm{n}=10)$ \\
\hline Gender & $64(80 \%)$ & $8(62 \%)$ & $5(50 \%)$ \\
Fale & $16(20 \%)$ & $5(38 \%)$ & $5(50 \%)$ \\
Ethnicity Caucasian & $7(90 \%)$ & $11(85 \%)$ & $\cdot$ \\
\hline Non-Caucasian & $8(10 \%)$ & $2(15 \%)$ & $\cdot$ \\
Mean age at explantation & $48.6 \pm 12.3$ & $47.0 \pm 11.6$ & $47.6 \pm 11.2$ \\
Presence of LVAD & $48(59 \%)$ & $8(62 \%)$ &.
\end{tabular}

$\mathrm{DCM}=$ dilated cardiomyopathy, $\mathrm{HCM}=$ hypertrophic cardiomyopathy, $\mathrm{LVAD}=$ left ventricular assist device.

\subsection{Identification of Rare Damaging Variants}

Results from the WES of all tissue samples showed a total of 1,788 rare damaging variants with the following distribution: 780 LoF, 1005 DMIS, and 3 CNVs.

When looking only at genes in the CMP panel, LoF variants were the most common, followed by DMIS variants (Table 4.2).

Table 4.2 Numbers of variants found in CMP genes in all tissue samples

\begin{tabular}{lcccccccc}
\hline Gene & LoF & DMIS & CNV (del) & & Gene & LoF & DMIS & CNV (del) \\
\hline TTN & 14 & 1 & 2 & & MYH7 & 0 & 2 & 0 \\
DSP & 3 & 1 & 0 & & SYNM & 0 & 2 & 0 \\
LMNA & 3 & 2 & 0 & & ALPK3 & 0 & 1 & 0 \\
MYBPC3 & 3 & 0 & 0 & & EYA4 & 0 & 1 & 0 \\
BAG3 & 2 & 0 & 0 & & FLNC & 0 & 1 & 1 \\
SYNE1 & 2 & 0 & 0 & & LAMA4 & 0 & 1 & 0 \\
ALMS1 & 1 & 0 & 0 & & PDLIM3 & 0 & 1 & 0 \\
SCN5A & 1 & 0 & 0 & & TBX20 & 0 & 1 & 0 \\
MYH6 & 0 & 2 & 0 & & TNNT2 & 0 & 1 & 0 \\
\hline
\end{tabular}

Genes highlighted in grey are definite dilated cardiomyopathy genes. Bolded genes are definite hypertrophic cardiomyopathy genes. LoF = Loss of function, DMIS = deleterious missense, $\mathrm{CNV}(\mathrm{del})=$ copy number variation deletion . 
Three samples contained two variants in CMP genes. The highest number of variants were in TTN, with 14 LoF, 1 DMIS and 2 CNV deletions.

\subsubsection{DCM hearts: LoF, DMIS and CNVs in CMP genes}

Pathogenic variants (LoF, DMIS or CNVs) in CMP genes were identified in 37 out of 80 DCM samples (46.3\%).

LoF variants in CMP genes in DCM hearts are shown in table 4.3. Ten of the 25 LoF variants in were present in ClinVar. Note that samples A0006 and A0023 have the exact same variant, as well as samples A0018 and A0168.

Table 4.3 DCM hearts: Loss of function variants in CMP genes

\begin{tabular}{llllc}
\hline ID & Gene & Variant & Impact & ClinVar \\
\hline A0053 & ALMS1 & Chr2:73679577delA & Frameshift(p.Glu1974fs) &. \\
A0018 & BAG3 & Chr10:121429549C>T & Nonsense(p.Arg123*) & P/L \\
A0168 & BAG3 & Chr10:121429549C>T & Nonsense(p.Arg123*) & P/L \\
A0043 & DSP & Chr6:7542319G>C & Splicing(.) &. \\
A0083 & DSP & Chr6:7579618C $>$ G & Nonsense(p.Tyr1065*) & P \\
A0158 & DSP & Chr6:7585261delTAAG & Frameshift(p.Ser2591fs) &. \\
A0096 & LMNA & Chr1:156104740G>T & Nonsense(p.Glu262*) & L \\
A0131 & LMNA & Chr1:156084806G>T & Nonsense(p.Glu33*) &. \\
A0169 & LMNA & Chr1:156106747delA & Frameshift(p.Asn473fs) &. \\
A0127 & MYBPC3 & Chr11:47373058T>C & Splicing(.) & P \\
A0112 & SCN5A & Chr3:38616882C>T & Nonsense(p.Trp1191*) &. \\
A0006 & TTN & Chr2:179602929dupCT & Frameshift(p.Ser4751fs) &. \\
A0023 & TTN & Chr2:179602929dupCT & Frameshift(p.Ser4751fs) &. \\
A0027 & TTN & Chr2:179422376G>C & Nonsense(p.Tyr29235*) &. \\
A0033 & TTN & Chr2:179494999delA & Frameshift(p.Asn14750fs) &. \\
A0052 & TTN & Chr2:179416848dupT & Frameshift(p.Tyr30260fs) & L \\
A0082 & TTN & Chr2:179439257G>A & Nonsense(p.Arg23868*) & P/L \\
A0087 & TTN & Chr2:179449558G>A & Nonsense(p.Arg21604*) &. \\
A0092 & TTN & Chr2:179484615C>T & Splicing(.) &. \\
A0102 & TTN & Chr2:179439467G>A & Nonsense(p.GIn23798*) &. \\
A0103 & TTN & Chr2:179456193delA & Frameshift(p.Gly20087fs) &. \\
A0132 & TTN & Chr2:179428823G>A & Nonsense(p.Gln27346*) & P/L \\
A0134 & TTN & Chr2:179426483G>A & Nonsense(p.Gln28126*) & L \\
A0138 & TTN & Chr2:179479481C>G & Splicing(.) & L \\
A0161 & TTN & Chr2:179432278delT & Frameshift(p.Ser26194fs) &. \\
\hline
\end{tabular}

Genes highlighted in grey are definite dilated cardiomyopathy genes. Bolded genes are definite hypertrophic cardiomyopathy genes. A dot (.) in the impact column indicates that the amino acid change is unknown. A dot in the ClinVar column indicates that the variant has not been submitted in ClinVar. P; Pathogenic, L; Likely pathogenic, ID: patient identification number. 
DMIS variants in CMP genes in DCM hearts are shown in table 4.4. Subject A0112 has both a LoF variant in SCN5A and a DMIS variant in PDLIM3.

Table 4.4 DCM hearts: Deleterious missense variants in CMP genes

\begin{tabular}{llllc}
\hline ID & Gene & Variant & Impact & ClinVar \\
\hline A0112 & PDLIM3 & Chr4:186423592G>C & Missense(p.Cys317Trp) & $\cdot$ \\
A0172 & EYA4 & Chr6:133836537C>T & Missense(p.Thr533lle) & $\cdot$ \\
A0020 & LMNA & Chr1:156084863C>G & Missense(p.Leu52Val) & L \\
A0141 & LMNA & Chr1:156104315G >T & Missense(p.Ser212lle) & $\cdot$ \\
A0110 & MYH6 & Chr14:23865525C>T & Missense(p.Met799lle) & $\cdot$ \\
A0009 & MYH7 & Chr14:23886457C>T & Missense(p.Arg1475His) & $\cdot$ \\
A0056 & MYH7 & Chr14:23898475C>A & Missense(p.Gly407Val) & CIP \\
A0045 & SYNM & Chr15:99671390C>T & Missense(p.Ser941Phe) & $\cdot$ \\
A0120 & SYNM & Chr15:99671372C>T & Missense(p.Ser935Phe) & $\cdot$ \\
A0147 & TBX20 & Chr7:35289588C>T & Missense(p.Glu119Lys) & $\cdot$ \\
A0061 & TTN & Chr2:179449216C>G & Missense(p.Gly21688Arg) &. \\
\hline
\end{tabular}

Genes highlighted in grey are definite dilated cardiomyopathy genes. Bolded genes are definite hypertrophic cardiomyopathy genes. A dot (.) in the impact column indicates that the amino acid change is unknown. A dot in the ClinVar column indicates that the variant has not been submitted in ClinVar. L; Likely pathogenic, CIP; Conflicting interpretation of pathogenicity, ID: patient identification number.

Copy number variations were only found in DCM hearts, see table 4.5. Notably, subject A0056 was also identified with a DMIS variant in MYH7. These CNVs are in exons with high PSI (percent spliced in) values, which means that they are likely to be spliced into the genome and there for a CNV in the exon, which is likely to have pathogenic effects on the protein.

Table 4.5 DCM hearts: Copy number variation deletions in CMP genes

\begin{tabular}{llll}
\hline ID & Gene & Genomic position & Phenotype \\
\hline A0021 & TTN & Chr2: 179614952- 179638238 & DCM \\
A0056 & TTN & Chr2: 179407763- 179460214 & DCM \\
A0165 & FLNC & Chr7: 128479982-128487866 & DCM
\end{tabular}

Genes highlighted in grey are definite dilated cardiomyopathy genes. DCM: Dilated cardiomyopathy, ID: Patients identification number.

\subsubsection{HCM hearts: LoF and DMIS Variants in CMP Genes}

LoF or DMIS variants in CMP genes were identified in 5 out of $13 \mathrm{HCM}$ samples (38.4\%). Three LoF variants were found in CMP genes in HCM hearts, see table 4.6. 
Table 4.6 HCM hearts: Loss of function variants in CMP genes

\begin{tabular}{llllc}
\hline ID & Gene & Variant & Impact & ClinVar \\
\hline A0076 & MYBPC3 & Chr11:47359280dupG & Frameshift(p.Trp792fs) & P \\
A0126 & MYBPC3 & Chr11:47360231C>G & Splicing(.) & . \\
A0070 & SYNE1 & Chr6:152639400T>C & Splicing(.) & . \\
\hline
\end{tabular}

Bold-printed genes are definite hypertrophic cardiomyopathy genes. A dot (.) in the impact column indicates that the amino acid change is unknown. A dot in the ClinVar column indicates that the variant has not been submitted in ClinVar. P; Pathogenic, ID:

Patient identification number.

Three DMIS variants were found in CMP genes in HCM hearts, see table 4.7. Subject A0126 has both a LoF variant in MYBPC3 and a DMIS variant in MYH6. No CNVs were found in HCM hearts.

Table 4.7 Deleterious missense variants in CMP genes in HCM patients

\begin{tabular}{llllc}
\hline ID & Gene & Variant & Impact & ClinVar \\
\hline A0126 & MYH6 & Chr14:23874506C $>$ T & Missense(p.Arg143GIn) & $\cdot$ \\
A0054 & ALPK3 & Chr15:85382216C>G & Missense(p.Pro306Ala) & $\cdot$ \\
A0108 & TNNT2 & Chr1:201334425C>T & Missense(p.Arg104GIn) & P/L \\
\hline
\end{tabular}

Boldfaced genes are definite hypertrophic cardiomyopathy genes. Genes highlighted in grey are definite dilated cardiomyopathy genes. A dot in the ClinVar column indicates that the variant has not been submitted to ClinVar. P/L:Pathogenic/Likely pathogenic. ID: Patient identification number.

\subsubsection{Control Donor Hearts: LoF and DMIS Variants in CMP Genes}

LoF or DMIS in CMP genes were identified in 3 out of 10 control donor hearts $(30.0 \%)$, see table 4.8. Sample H0009 had two DMIS variants, one in DSP and the other in FLNC. Note that DSP is the only gene that is marked as a definite CMP gene in the CMP gene panel (Figure 3.1). No CNVs were found in control hearts.

Table 4.8 Control donor hearts with LoF or DMIS variants in CMP genes

\begin{tabular}{lllllc}
\hline ID & LoF/DMIS & Gene & Variant & Impact & ClinVar? \\
\hline H0036 & LoF & SYNE1 & Chr6:152949399C>A & Splicing(.) & $\cdot$ \\
H0009 & DMIS & DSP & Chr6:7569482G>A & Missense(p.Val495Met) & US \\
& DMIS & FLNC & Chr7:128494151G>A & Missense(p.Arg2203His) & . \\
H0035 & DMIS & LAMA4 & Chr6:112451225T>C & Missense(p.Asp1329Gly) & US \\
\hline
\end{tabular}

Boldfaced genes are definite hypertrophic cardiomyopathy genes. A dot (.) in the impact column indicates that the amino acid change is unknown. A dot in the ClinVar column indicates that the variant has not been submitted to ClinVar. US: Unknown significance.

ID: Patient identification number. 


\subsection{Confirmation of Variants}

All LoF and DMIS variants in CMP genes were confirmed by looking into IGV for each sample at the position of the variant. Figure 4.1 is an example of IGV confirmation of a LoF variant in DSP in sample A0043.

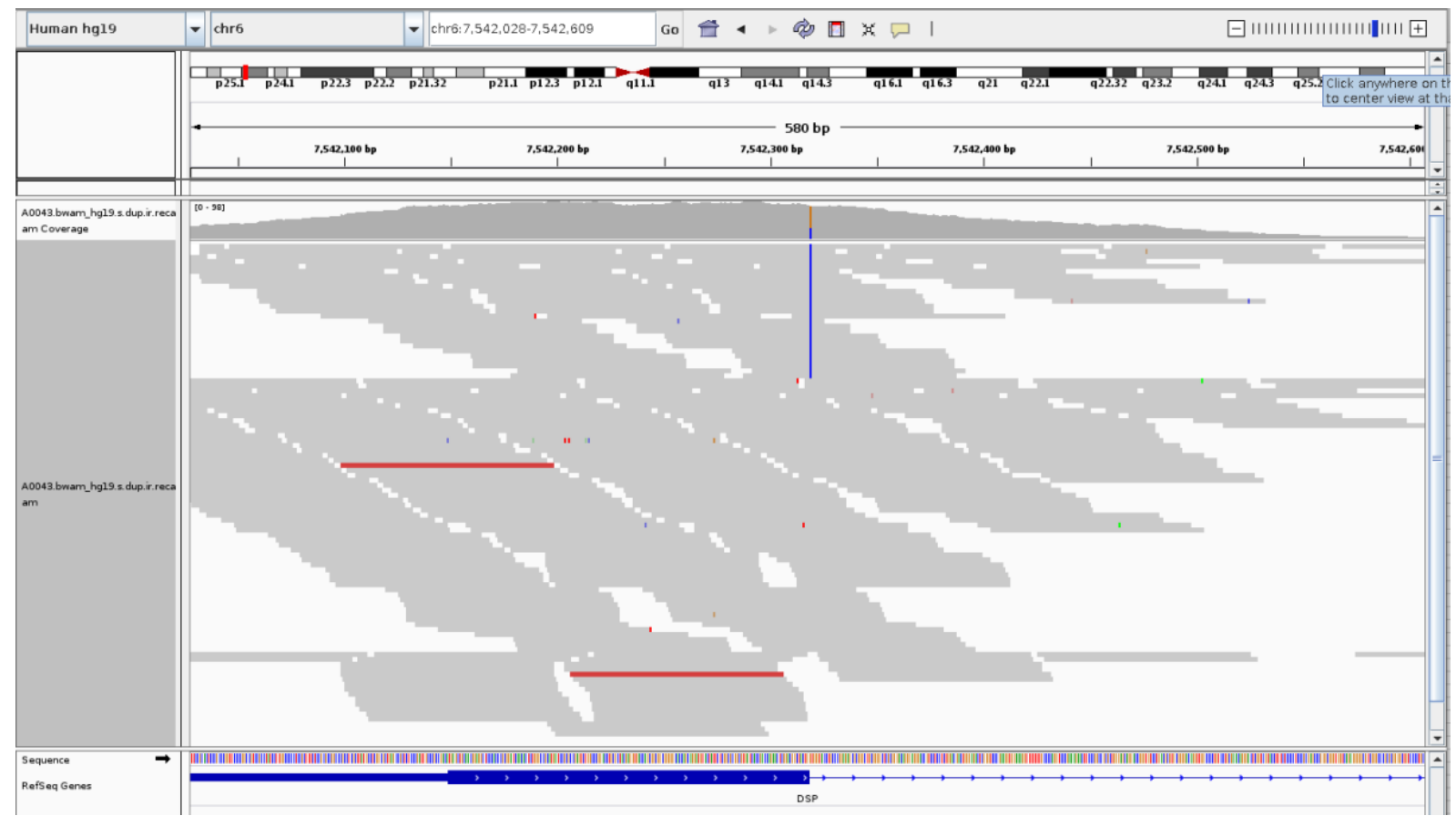

Figure 4.1 IGV confirmation of a loss of function variant

The figure shows the heterozygous loss of function variant in $D S P(\mathrm{Chr6:7542319G>C})$ in sample A0043.

Furthermore, all LoF variants identified in CMP genes were successfully confirmed by Sanger sequencing. Figure 4.2 exemplifies a variant confirmation by Sanger sequencing.

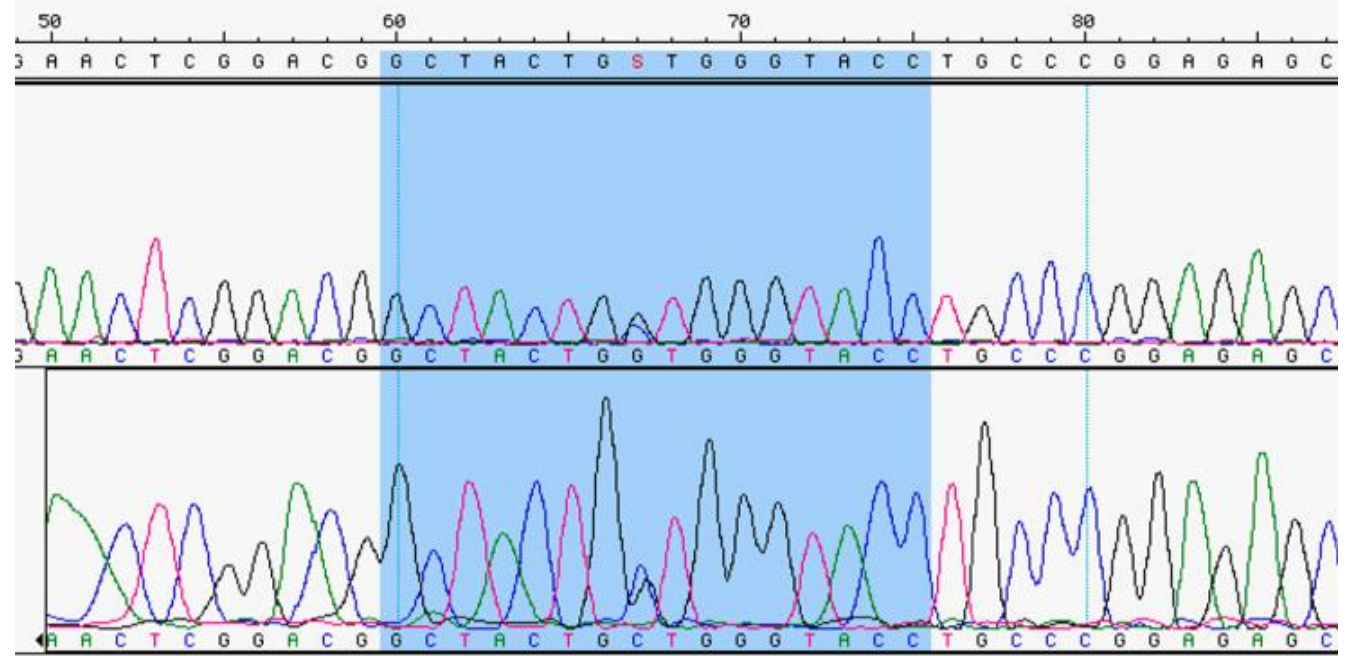

Figure 4.2 Confirmation of a loss of function variant by Sanger sequencing

The figure shows the Sanger sequencing results of sample A0043 which had a loss of function variant (Chr6:7542319G $>C$ ) in $D S P$. In the middle of the blue highlighted area, the change from $G$ to $C$ can be seen. 
CNV deletions were examined by RNA-seq and the results showed a decrease in RNA expression from the region of the deletions in TTN in samples A0021 and A0056 but no change in the RNA expression from the region of the estimated deletion in FLNC in sample A0165. This indicates that the two deletions in TTN in samples A0021 and A0056 respectively are truly present and affect RNA expression. The deletion initially detected in FLNC in sample A0165 is unlikely to be the cause of the disease.

\subsection{Non-CMP Genes With Enriched Frequency of Variants}

The results of the burden analyses showed that four genes that were not in the CMP panel had an enriched frequency of loss of function variants compared to the reference population with genomewide significance ( $\mathrm{p} \leq 2.5 \mathrm{E}-06$ ), see table 4.9 . Note that $L E K R 1$ is not highly expressed in the heart like MDK, UBE2I and MATR3 are. No DMIS variants were over-represented in genes in the study cohort compared to gnomAD.

Table 4.9 Non-CMP genes with enriched frequency of LoF variants

\begin{tabular}{lccc}
\hline & $\begin{array}{c}\text { DCM } \\
(\mathrm{n}=80)\end{array}$ & $\begin{array}{c}\text { GnomAD } \\
(\mathrm{n}=141,456)\end{array}$ & $\begin{array}{c}\text { DCM vs. } \\
\text { GnomAD }\end{array}$ \\
\hline & Variants $(\mathrm{n})$ & Variants $(\mathrm{n})$ & $\mathrm{p}$-value \\
\hline MDK* & 2 & 2 & $2.35 \mathrm{E}-06$ \\
LEKR1 $^{*}$ & 2 & 2 & $1.04 \mathrm{E}-06$ \\
UBE2 I $^{*}$ & 1 & 0 & $2.2 \mathrm{E}-16$ \\
MATR3 $^{*}$ & 1 & 0 & $2.2 \mathrm{E}-16$ \\
\hline
\end{tabular}

DCM; Dilated cardiomyopathy, HCM; Hypertrophic cardiomyopathy, GnomAD; Genome Aggregation Database.

*HHE genes.

The details of the enriched loss of function variants in non-CMP genes are presented in table 4.10. They were all in DCM subjects. None of them has been annotated in ClinVar.

Table 4.10 Loss of function variants in non-CMP genes with enriched frequency of variants

\begin{tabular}{|c|c|c|c|c|}
\hline ID & Gene & Variant & Impact & ClinVar \\
\hline A0109 & $M D K^{*}$ & Chr11:46404340insGGGGGGGGGGGGGC & Frameshift(p.Ala150fs) & . \\
\hline A0099 & $M D K^{*}$ & Chr11:46404340insGGGGGGGGGGGGGC & Frameshift(p.Ala150fs) & . \\
\hline A0090 & UBE2I* & Chr16:1370586delGGGAG & Frameshift(p.Arg161fs) & . \\
\hline A0090 & UBE21* & Chr16:1370592insA & Frameshift(p.Trp163fs) & . \\
\hline A0073 & MATR3* & $\begin{array}{l}\text { Chr5:138658194delGTAATTTCATTTTGTTTT } \\
\text { TCATATGTGTGAGTATATTCAACTTTACTTTT } \\
\text { TCAGACAACAAATTAATTGTGGTGTGTCCTT } \\
\text { TTGATTTCAG }\end{array}$ & Splicing(.) & . \\
\hline A0033 & LEKR1 & Chr3:156645283dupGGAA & Frameshift(p.Leu152fs) & . \\
\hline A0061 & LEKR1 & Chr3:156742600G >T & Nonsense(p.Glu419*) & . \\
\hline
\end{tabular}

* $=$ HHE genes. A dot (.) in the impact column indicates that the amino acid change is unknown. A dot in the ClinVar column indicates that the variant has not been annotated in ClinVar.

ID: Patient identification number. 


\section{Discussions}

This study aimed to identify genetic variants in end-stage cardiomyopathy patients. A pathogenic variant (LoF, DMIS, or CNV deletion) in genes on the CMP gene panel was identified in 45 of all 103 samples (43.7\%) with the following distribution: 37 out of 80 DCM samples (46.3\%), 5 out of $13 \mathrm{HCM}$ samples (38.4\%), and 3 out of 10 control samples (30.0\%).

Additionally, binomial significant LoF variants in HHE genes were identified in the genes $M D K$, UBE2I, and MATR3 in 5 samples, all DCM (5.0\% of the DCM cohort).

Two DCM cases were found with genome-wide significant LoF variants in the gene LEKR1, which is in neither of the gene panels (2.5\% of the DCM cohort).

Taken together these analyses identified 52 samples with potentially pathogenic variants (50.5\% of the whole cohort).

\subsection{Assessment of Variant Pathogenicity}

The study cohort was special in the way that the cardiomyopathy patients were all in the end stage of their disease when the samples were collected. The high proportion of pathogenic variants in the study cohort raises the question if end-stage cardiomyopathy patients are more prone to genetic causes for their disease than other cardiomyopathy patients. Furthermore, it would be interesting to know if specific mutations are more related to the pathogenesis of end-stage cardiomyopathy than others.

Another reason for the high prevalence of variants detected in the cohort might be that, unlike most previous genetic studies on cardiomyopathy, cardiac tissue was studied, but not blood. Mosaic individuals have two or more genotypically distinct cell lines derived from a single zygote. That means different cell lines may be present in various tissues.(79) The genetic testing of tissue, therefore, can detect mosaic variants in the DNA of cells in the specific tissue that may not be present in the blood. It would be interesting to further investigate the variants detected in this study to evaluate if they are mosaic.

Generally, loss of function variants are more pathogenic than missense variants. Loss of function variants cause a complete loss of function of the protein that the gene encodes. Missense variants cause the substitution of one amino acid for another. Hence, their pathogenicity is more difficult to interpret. $(80,81)$ Even though the filtration of variants in this study only selected for deleterious missense variants, further functional testing must confirm the effect of the variants on the organism.

\subsubsection{Pathogenic Variants in DCM Patients}

Of the 7 CMP genes with detected LoF variants, TTN, DSP, and LMNA are definite DCM genes.(74) MYBPC3, BAG3, ALMS1 and SCN5A are not as definite DCM genes.

$M Y B P C 3$ is a primarily a definite HCM gene, but it has also been correlated with the pathogenesis of DCM. $(82,83) B A G 3$ encodes an antiapoptotic protein in the Z-disc of the sarcomere, and variants in it have been correlated with the pathogenesis of DCM. Relatively few cases of DCM patients with variants in BAG3 have been reported, but male sex, decreased LV EF, and enlarged enddiastolic diameter have been associated with adverse outcomes in those patients.(84) The variants in 
BAG3 are in the exact same location in the two patients carrying BAG3 variants, indicating that these two patients may be related; in addition, both are males. ALMS1 encodes a large protein found in the cytosol, centrosomes, and basal bodies of cells. Defects in the gene are known to cause Alström syndrome, an autosomal recessive disease characterized by multiple defects, including cardiomyopathy.(85) SCN5A has been implicated in the pathogenesis of DCM for a long time, even though previous studies revealed there is no excess variation in DCM cases compared to reference genomes.(74) Samples A0006 and A0023 have the exact same frameshift variant in TTN. Further clinical information about the patients would be helpful to discover whether these two individuals are related.

Compared to the LoF variants, the proportion of DMIS variants found in putative DCM genes was higher, and the proportion of variants submitted to ClinVar as pathogenic or likely pathogenic was lower. This is consistent with the fact that the pathogenicity of DMIS variants is more difficult to interpret than LoF variants.

DMIS variants were found in 8 CMP genes. There, three of them are definite DCM genes ( TTN, $M Y H 7$, and $L M N A$ ), while the correlation of the other genes (PDLIM3, EYA4, MYH6, SYNM, and TBX2O) with the pathogenicity of DMC is not as clear.(74)

LoF variants in TTN have a stronger correlation with the pathogenesis of DCM than DMIS variants, while the opposite applies to MYH7. LoF and DMIS variants in LMNA have been almost equally associated with the pathogenesis of DCM.(74)

$P D L I M 3$ encodes a protein in the Z-line in the sarcomere, but the mechanism behind the correlation between variants in PDLIM3 and the DCM phenotype is unclear.(86) EYA4 encodes a transcriptional coactivator in which substantial deletions have been correlated with the development of DCM preceded by sensorineural hearing loss.(87) The consequences of DMIS variants in EYA4 are not well established. Few DMIS variants in MYH6 have been reported in DCM patients, but they have been associated with phenotypes of both DCM and HCM.(88) The same goes for SYNM, which encodes the protein Synemin. This protein stabilizes intercalated discs in sarcomeres. $(89,90)$ Variants in TBX20 (the T-box family transcription factor gene) have been shown to be associated with the adult onset of DCM.(91)

Three DCM samples were identified with CNV deletions initially. The RNA-seq results revealed that the two deletions in TTN in samples A0021 and A0056, respectively, affect RNA expression and are, thus, truly present in the genome of those patients. The deletion initially detected in FLNC in sample A0165 is unlikely to cause disease since the RNA-seq results showed no decrease in RNA expression around the potential deletion.

According to these results, LoF, DMIS, and CNV variants in TTN are likely to be the genetic cause of the disease in $21.3 \%$ of the DCM cohort in this study, which is a higher proportion than previous studies have established.(14)

\subsubsection{Pathogenic Variants in HCM Patients}

Of the $3 \mathrm{LoF}$ variants detected in CMP genes in the HCM cohort, 2 were in the definite HCM gene MYBPC3; the one in sample A0076 is annotated as pathogenic in ClinVar. The other was in SYNE1. SYNE1 encodes Nespirin-1, which is a protein in the outer nuclear membrane of cells.(92) 
SYNE1 is in the CMP gene panel, but later studies have demonstrated that variants in the gene do not appear to cause disease.(74)

Three DMIS variants in the genes MYH6, ALPK3, and TNNT2 were discovered in the HCM cohort as well. TNNT2 is a definite HCM gene; in a 2016 study of 180 families with genetically caused cardiomyopathies, $11.7 \%$ were identified with mutations in TNNT2.(93) Truncating variants in ALPK3 have been correlated with severe pediatric cardiomyopathy (94), while less is known about the severity of DMIS variants in the gene. The variant in MYH6 was discovered in sample A0126, which was detected with an LoF variant in MYBPC3 as well. The variant in MYBPC3 is more likely to be the cause of disease in A0126, since MYBPC3 is a definite HCM gene, while the pathogenicity of variants in MYH6 is less clear.

\subsubsection{Pathogenic Variants in Control Hearts}

Interestingly, $30 \%$ of the controls were identified with damaging genomic variants. These results are surprising since the control hearts were not known to have a clinical diagnosis of cardiomyopathy. Nevertheless, the control hearts were potential donor hearts that, for reasons unknown at the time of this study, did not get implanted. Accordingly, some functional effects from these defects may have been detected in these donor hearts that hypothetically may have prevented implantation. In addition, when looking further into the variants, there is evidence that the likelihood decreases that these variants are pathogenic.

In sample H0009, two DMIS variants were detected in genes from the CMP gene panel. The variant in $D S P$ is annotated in ClinVar as a variant of unknown significance. Loss of function variants in $D S P$ are strongly correlated with the pathogenesis of $D C M$, but the significance of missense variants in $D S P$ causing DCM is not as well established. (74) This casts doubt upon the pathogenicity of the variant. The variant in FLNC in H0009 has not been annotated in ClinVar, and FLNC is not a definite CMP gene, even though it has been correlated with the pathogenesis of cardiomyopathy. $(95,96)$

In sample H0035, a DMIS variant in LAMA4 was discovered. The variant had been annotated in ClinVar as a variant of unknown significance, which makes it hard to interpret its pathogenicity.

In sample H0036, a loss-of-function variant in the gene SYNE1 was found that has not been annotated as pathogenic in ClinVar. As mentioned above, the pathogenicity of variants in SYNE1 is unclear. More clinical information about the condition of the donor hearts is required for further interpretation of these results.

\subsection{Interpretation of Burden Analysis}

Of the 780 LoF and 1,005 DMIS variants found in genes in the cohort, only a fraction was present in genes from the CMP panel. The significance of the pathogenicity of variants in genes that are not in the CMP gene panel was estimated by burden analyses since genes that have the frequency of variants in them enriched compared to the frequency of variants found in the same genes in a healthy reference cohort are likely to have something to do with the pathogenesis of the disease. The binomial test provided information about the likelihood of the discovery of variants in genes being coincidental. The estimated p-value 2.5E-06 is as low as it is because of the multi-testing correction of 20,000 genes. The variants shown not to be enriched in the study population compared to gnomAD are still in place in 
the patient genome and cannot be excluded from causing disease without further functional testing and analyses.

The HHE genes MDK, UBE2I, and MATR3 were found to have an enriched frequency of pathogenic variants compared to gnomAD. MDK encodes the heparin binding growth factor midkine, which is expressed in various cell types during embryogenesis. Hypoxia increases the expression of $M D K$, which has shown to enhance the expression of myocardin, which directs smooth muscle differentiation. This leads to pulmonary vascular remodeling.(97) It would be interesting to investigate the effect of increased expression of $M D K$ on the myocardium. UBE2/ encodes a ubiquitin-conjugating enzyme that takes part in the selective degradation of proteins.(98) Investigation of the function of the enzyme in the heart when a pathogenic variant is present in the gene could possibly reveal pathogenicity's mechanism. Hypothetically, a variated UBE2I gene could cause abnormality in the degradation of proteins in the myocardium. MATR3 encodes an RNA- and DNA-binding protein, and variants in the gene can cause the pathogenesis of distal myopathy. $(99,100)$ This raises the question if variants in MATR3 could also participate in the development of cardiomyopathy.

The gene LEKR1, which is in neither in the CMP gene panel nor a HHE gene, was also found to have an enriched frequency of pathogenic variants. As this gene is not highly expressed in the heart, further investigation and functional testing are needed to properly interpret the pathogenicity of variants in this gene.

\subsection{Limitations}

The study cohort is small, which affects the study's statistical power. WES only provides information about the exons in the genome. Therefore, variants in the introns of the genome cannot be found by WES.

The lack of ideal control samples limited the chance to compare cardiomyopathy patients with healthy hearts. The 10 control tissues were from people who had already died, and something hindered the hearts from being transplanted to donors. This lack of information about the control hearts makes it hard to know if the hearts were really healthy. Therefore, it is difficult to interpret the consequences of variants found in their genome.

Lack of information about the clinical presentation of the disease in the cardiomyopathy patients made it impossible to interpret if variants in specific genes were more correlated to a specific clinical presentation.

\subsection{Conclusions}

Compared to previous studies that analyze DNA from peripheral blood samples, the proportion of deleterious genomic variants in cardiac tissue in the DCM cohort of this study was substantially higher. This might indicate that end-stage DCM patients are more likely to have pathogenic variants in their genome that cause their disease. It also raises the question if possibly more variants are found by sequencing DNA extracted from tissue versus blood.

As discussed in the introduction, WES and WGS very often lead to the findings of VUS since variants are searched for in all the genes of the genome. The results from this study were no exception, but computational methods and analyses were used to interpret the pathogenicity of the variants found 
in the cohort. More clinical information about all the patients in the study cohort would allow further research and comparison of genomic variants and clinical signs. 


\section{References}

1. Maron BJ, Towbin JA, Thiene G, Antzelevitch C, Corrado D, Arnett D, et al. Contemporary definitions and classification of the cardiomyopathies: an American Heart Association Scientific Statement from the Council on Clinical Cardiology, Heart Failure and Transplantation Committee; Quality of Care and Outcomes Research and Functional Genomics and Translational Biology Interdisciplinary Working Groups; and Council on Epidemiology and Prevention. Circulation. 2006;113(14):1807-16.

2. Cecchi F, Tomberli B, Olivotto I. Clinical and molecular classification of cardiomyopathies. Global Cardiology Science \& Practice. 2012;2012(1):4.

3. Lakdawala NK. Using genetic testing to guide therapeutic decisions in cardiomyopathy. Current treatment options in cardiovascular medicine. 2013;15(4):387-96.

4. Rehman I, Rehman A. Anatomy, Thorax, Heart Left Anterior Descending (LAD) Artery. StatPearls [Internet]: StatPearls Publishing; 2018.

5. Home Page: National Library of Medicine (US). Genetics home reference [Internet] Bethesda (MD): The Library; 2019 [cited 2019 April 9]. Available from: https://ghr.nlm.nih.gov/gallery?start=40.

6. Seidman J, Seidman C. The genetic basis for cardiomyopathy: from mutation identification to mechanistic paradigms. Cell. 2001;104(4):557-67.

7. $\quad$ Roger VL. Epidemiology of heart failure. Circulation research. 2013;113(6):646-59.

8. Metra M, Teerlink JR. Seminar Heart failure. The Lancet. 2017;390(10106):1981-95.

9. Hartupee J, Mann DL. Neurohormonal activation in heart failure with reduced ejection fraction. Nature Reviews Cardiology. 2017;14(1):30.

10. Florea VG, Cohn JN. The autonomic nervous system and heart failure. Circulation research. 2014;114(11):1815-26.

11. He BJ, Anderson ME. Aldosterone and cardiovascular disease: the heart of the matter. Trends in Endocrinology \& Metabolism. 2013;24(1):21-30.

12. Kumar S, Stevenson WG, John RM. Arrhythmias in dilated cardiomyopathy. Cardiac electrophysiology clinics. 2015;7(2):221-33.

13. Jefferies JL, Towbin J. Dilated cardiomyopathy. The Lancet. 2010;375(9716):752-62.

14. Burke MA, Cook SA, Seidman JG, Seidman CE. Clinical and Mechanistic Insights Into the Genetics of Cardiomyopathy. Journal of the American College of Cardiology. 2016;68(25):2871-86. 15. Elliott P, Andersson B, Arbustini E, Bilinska Z, Cecchi F, Charron P, et al. Classification of the cardiomyopathies: a position statement from the European Society Of Cardiology Working Group on Myocardial and Pericardial Diseases. European heart journal. 2008;29(2):270-6.

16. Tardiff JC, Carrier L, Bers DM, Poggesi C, Ferrantini C, Coppini R, et al. Targets for therapy in sarcomeric cardiomyopathies. Cardiovascular research. 2015;105(4):457-70.

17. Garfinkel AC, Seidman JG, Seidman CE. Genetic Pathogenesis of Hypertrophic and Dilated Cardiomyopathy. Heart failure clinics. 2018;14(2):139-46.

18. Alamo L, Ware JS, Pinto A, Gillilan RE, Seidman JG, Seidman CE, et al. Effects of myosin variants on interacting-heads motif explain distinct hypertrophic and dilated cardiomyopathy phenotypes. eLife. 2017;6:e24634.

19. Zoghbi ME, Woodhead JL, Moss RL, Craig R. Three-dimensional structure of vertebrate cardiac muscle myosin filaments. Proceedings of the National Academy of Sciences.

2008;105(7):2386-90.

20. McNally EM, Mestroni L. Dilated Cardiomyopathy: Genetic Determinants and Mechanisms. Circulation research. 2017;121(7):731-48.

21. Yin Z, Ren J, Guo W. Sarcomeric protein isoform transitions in cardiac muscle: a journey to heart failure. Biochimica et Biophysica Acta. 2015;1852(1):47-52.

22. LeWinter MM, Granzier HL. Cardiac titin and heart disease. Journal of cardiovascular pharmacology. 2014;63(3):207-12.

23. Squire JM. Muscle contraction: Sliding filament history, sarcomere dynamics and the two Huxleys. Global Cardiology Science \& Practice. 2016;2016(2):e201611.

24. Taylor MR, Carniel E, Mestroni L. Cardiomyopathy, familial dilated. Orphanet journal of rare diseases. 2006;1(1):27.

25. McKenna WJ, Maron BJ, Thiene G. Classification, epidemiology, and global burden of cardiomyopathies. Circulation research. 2017;121(7):722-30.

26. Weintraub RG, Semsarian C, Macdonald P. Dilated cardiomyopathy. Lancet (London, England). 2017;390(10092):400-14. 
27. Tripathi R, Sullivan R, Fan T-HM, Wang D, Sun Y, Reed GL, et al. Enhanced heart failure, mortality and renin activation in female mice with experimental dilated cardiomyopathy. PloS one. 2017;12(12):e0189315.

28. Miller AB. Aldosterone antagonism in heart failure. Vascular health \& risk management. 2007;3(5):605-9.

29. Mestroni L, Maisch B, McKenna WJ, Schwartz K, Charron P, Rocco C, et al. Guidelines for the study of familial dilated cardiomyopathies. Collaborative Research Group of the European Human and Capital Mobility Project on Familial Dilated Cardiomyopathy. European heart journal. 1999;20(2):93-102.

30. Assomull RG, Prasad SK, Lyne J, Smith G, Burman ED, Khan M, et al. Cardiovascular magnetic resonance, fibrosis, and prognosis in dilated cardiomyopathy. Journal of the American College of Cardiology. 2006;48(10):1977-85.

31. McMurray JJ, Packer M, Desai AS, Gong J, Lefkowitz MP, Rizkala AR, et al. Angiotensinneprilysin inhibition versus enalapril in heart failure. New England Journal of Medicine.

2014;371(11):993-1004.

32. Bardy GH, Lee KL, Mark DB, Poole JE, Packer DL, Boineau R, et al. Amiodarone or an implantable cardioverter-defibrillator for congestive heart failure. New England Journal of Medicine. 2005;352(3):225-37.

33. Topilsky Y, Pereira NL, Shah DK, Boilson B, Schirger JA, Kushwaha SS, et al. Left ventricular assist device therapy in patients with restrictive and hypertrophic cardiomyopathy. Circulation: Heart Failure. 2011;4(3):266-75.

34. Maron BJ, Maron MS. Hypertrophic cardiomyopathy. Lancet (London, England). 2013;381(9862):242-55.

35. Maron BJ. Clinical Course and Management of Hypertrophic Cardiomyopathy. The New England journal of medicine. 2018;379(7):655-68.

36. Pantazis A, Vischer AS, Perez-Tome MC, Castelletti S. Diagnosis and management of hypertrophic cardiomyopathy. Echo research and practice. 2015;2(1):R45-R53.

37. Cirino AL, Ho C. Hypertrophic cardiomyopathy overview. Gene Reviews. 2014.

38. Rahmani G, Kraushaar G, Dehghani P. Diagnosing burned-out hypertrophic cardiomyopathy: Daughter's phenotype solidifies father's diagnosis. Journal of cardiology cases. 2015;11(3):78-80.

39. Teekakirikul P, Kelly MA, Rehm HL, Lakdawala NK, Funke BH. Inherited cardiomyopathies: molecular genetics and clinical genetic testing in the postgenomic era. The Journal of molecular diagnostics : JMD. 2013;15(2):158-70.

40. Tejado BSM, Jou C. Histopathology in HCM. Global cardiology science \& practice. 2018;2018(3).

41. Raut M, Maheshwari A, Swain B. Awareness of 'systolic anterior motion'in different conditions. Clinical Medicine Insights: Cardiology. 2018;12:1179546817751921.

42. Vakrou S, Abraham MR. Hypertrophic cardiomyopathy: a heart in need of an energy bar? Frontiers in physiology. 2014;5:309.

43. Richter K, Kietzmann T. Reactive oxygen species and fibrosis: further evidence of a significant liaison. Cell and tissue research. 2016;365(3):591-605.

44. Raman B, Ariga R, Spartera M, Sivalokanathan S, Chan K, Dass S, et al. Progression of myocardial fibrosis in hypertrophic cardiomyopathy: mechanisms and clinical implications. European Heart Journal-Cardiovascular Imaging. 2018;20(2):157-67.

45. Todiere G, Aquaro GD, Piaggi P, Formisano F, Barison A, Masci PG, et al. Progression of myocardial fibrosis assessed with cardiac magnetic resonance in hypertrophic cardiomyopathy. Journal of the American College of Cardiology. 2012;60(10):922-9.

46. Elliott $\mathrm{P}$, Anastasakis A, Borger M, Borggrefe M, Cecchi F, Charron P. The task force for the diagnosis and management of hypertrophic cardiomyopathy of the European Society of Cardiology (ESC). European heart journal. 2014;35:2733-79.

47. Dunn KE, Caleshu C, Cirino AL, Ho CY, Ashley EA. A clinical approach to inherited hypertrophy: the use of family history in diagnosis, risk assessment, and management. Circulation: Cardiovascular Genetics. 2013;6(1):118-31.

48. Siontis KC, Geske JB, Ong K, Nishimura RA, Ommen SR, Gersh BJ. Atrial fibrillation in hypertrophic cardiomyopathy: prevalence, clinical correlations, and mortality in a large high-risk population. Journal of the American Heart Association. 2014;3(3):e001002.

49. Pelliccia F, Pasceri V, Limongelli G, Autore C, Basso C, Corrado D, et al. Long-term outcome of nonobstructive versus obstructive hypertrophic cardiomyopathy: a systematic review and metaanalysis. International journal of cardiology. 2017;243:379-84. 
50. Maron MS, Olivotto I, Zenovich AG, Link MS, Pandian NG, Kuvin JT, et al. Hypertrophic cardiomyopathy is predominantly a disease of left ventricular outflow tract obstruction. Circulation. 2006;114(21):2232-9.

51. Maron BJ, Maron MS, Wigle ED, Braunwald E. The 50-year history, controversy, and clinical implications of left ventricular outflow tract obstruction in hypertrophic cardiomyopathy: from idiopathic hypertrophic subaortic stenosis to hypertrophic cardiomyopathy. Journal of the American College of Cardiology. 2009;54(3):191-200.

52. Gersh BJ, Maron BJ, Bonow RO, Dearani JA, Fifer MA, Link MS, et al. 2011 ACCF/AHA Guideline for the Diagnosis and Treatment of Hypertrophic Cardiomyopathy: a report of the American College of Cardiology Foundation/American Heart Association Task Force on Practice Guidelines. Developed in collaboration with the American Association for Thoracic Surgery, American Society of Echocardiography, American Society of Nuclear Cardiology, Heart Failure Society of America, Heart Rhythm Society, Society for Cardiovascular Angiography and Interventions, and Society of Thoracic Surgeons. Journal of the American College of Cardiology. 2011;58(25):e212-60.

53. Patel SR, Saeed O, Naftel D, Myers S, Kirklin J, Jorde UP, et al. Outcomes of restrictive and hypertrophic cardiomyopathies after LVAD: an INTERMACS analysis. Journal of cardiac failure. 2017;23(12):859-67.

54. Watson JD, Crick FH. Molecular structure of nucleic acids. Nature. 1953;171(4356):737-8.

55. Heather JM, Chain B. The sequence of sequencers: The history of sequencing DNA.

Genomics. 2016;107(1):1-8.

56. Mogensen J, van Tintelen JP, Fokstuen S, Elliott P, van Langen IM, Meder B, et al. The current role of next-generation DNA sequencing in routine care of patients with hereditary cardiovascular conditions: a viewpoint paper of the European Society of Cardiology working group on myocardial and pericardial diseases and members of the European Society of Human Genetics. European heart journal. 2015;36(22):1367-70.

57. Slatko BE, Gardner AF, Ausubel FM. Overview of Next-Generation Sequencing Technologies. Current protocols in molecular biology. 2018;122(1):e59.

58. Bumgarner R. Overview of DNA microarrays: types, applications, and their future. Current protocols in molecular biology. 2013;101(1):22.1.1-.1. 11.

59. Lu Y, Shen Y, Warren W, Walter R. Next Generation Sequencing in Aquatic Models. Next Generation Sequencing-Advances, Applications and Challenges: IntechOpen; 2016.

60. van El CG, Cornel MC, Borry P, Hastings RJ, Fellmann F, Hodgson SV, et al. Whole-genome sequencing in health care: recommendations of the European Society of Human Genetics. European journal of human genetics : EJHG. 2013;21(6):580-4.

61. Alfares AA, Kelly MA, McDermott G, Funke BH, Lebo MS, Baxter SB, et al. Results of clinical genetic testing of 2,912 probands with hypertrophic cardiomyopathy: expanded panels offer limited additional sensitivity. Genetics in medicine : official journal of the American College of Medical Genetics. 2015;17(11):880-8.

62. Kukurba KR, Montgomery SB. RNA sequencing and analysis. Cold Spring Harbor Protocols. 2015;2015(11):951-69.

63. Bozkurt B, Colvin M, Cook J, Cooper LT, Deswal A, Fonarow GC, et al. Current Diagnostic and Treatment Strategies for Specific Dilated Cardiomyopathies: A Scientific Statement From the American Heart Association. Circulation. 2016;134(23):e579-e646.

64. Pinto YM, Elliott PM, Arbustini E, Adler Y, Anastasakis A, Bohm M, et al. Proposal for a revised definition of dilated cardiomyopathy, hypokinetic non-dilated cardiomyopathy, and its implications for clinical practice: a position statement of the ESC working group on myocardial and pericardial diseases. European heart journal. 2016;37(23):1850-8.

65. Morita H, Rehm HL, Menesses A, McDonough B, Roberts AE, Kucherlapati R, et al. Shared genetic causes of cardiac hypertrophy in children and adults. The New England journal of medicine. 2008;358(18):1899-908.

66. $\quad \mathrm{Li} \mathrm{H}$, Durbin R. Fast and accurate short read alignment with Burrows-Wheeler transform. Bioinformatics. 2009;25(14):1754-60.

67. Van der Auwera GA, Carneiro MO, Hartl C, Poplin R, Del Angel G, Levy-Moonshine A, et al. From FastQ data to high-confidence variant calls: the genome analysis toolkit best practices pipeline. Current protocols in bioinformatics. 2013;43(1):11.0. 1-.0. 33.

68. McKenna A, Hanna M, Banks E, Sivachenko A, Cibulskis K, Kernytsky A, et al. The Genome Analysis Toolkit: a MapReduce framework for analyzing next-generation DNA sequencing data. Genome research. 2010;20(9):1297-303.

69. Liu X, Jian X, Boerwinkle E. dbNSFP v2. 0: a database of human non-synonymous SNVs and their functional predictions and annotations. Human mutation. 2013;34(9):E2393-E402. 
70. Karczewski KJ, Francioli LC, Tiao G, Cummings BB, Alföldi J, Wang Q, et al. Variation across 141,456 human exomes and genomes reveals the spectrum of loss-of-function intolerance across human protein-coding genes. BioRxiv. 2019:531210.

71. Fromer M, Purcell SM. Using XHMM software to detect copy number variation in whole-exome sequencing data. Current protocols in human genetics. 2014;81(1):7.23. 1-7.. 1.

72. Ruderfer DM, Hamamsy T, Lek M, Karczewski KJ, Kavanagh D, Samocha KE, et al. Patterns of genic intolerance of rare copy number variation in 59,898 human exomes. Nature genetics. 2016;48(10):1107-011.

73. Garcia-Pavia P, Kim Y, Alejandra Restrepo-Cordoba M, Lunde IG, Wakimoto H, Smith AM, et al. Genetic Variants Associated with Cancer Therapy-Induced Cardiomyopathy. Circulation. 2019.

74. Walsh R, Thomson KL, Ware JS, Funke BH, Woodley J, McGuire KJ, et al. Reassessment of Mendelian gene pathogenicity using 7,855 cardiomyopathy cases and 60,706 reference samples. Genetics in Medicine. 2017;19(2):192-203.

75. Zaidi S, Choi M, Wakimoto H, Ma L, Jiang J, Overton JD, et al. De novo mutations in histonemodifying genes in congenital heart disease. Nature. 2013;498(7453):220-3.

76. Thorvaldsdóttir H, Robinson JT, Mesirov JP. Integrative Genomics Viewer (IGV): highperformance genomics data visualization and exploration. Briefings in bioinformatics. 2013;14(2):17892.

77. Wong K-F, Wong W-K, Lin M-S. Forward selection two sample binomial test. Journal of data science: JDS. 2014;12(4):279.

78. Untergasser A, Cutcutache I, Koressaar T, Ye J, Faircloth BC, Remm M, et al. Primer3-new capabilities and interfaces. Nucleic acids research. 2012;40(15):e115-e.

79. Gajecka M. Unrevealed mosaicism in the next-generation sequencing era. Molecular Genetics and Genomics. 2016;291(2):513-30.

80. Lodish H, Berk A, Zipursky SL, Matsudaira P, Baltimore D, Darnell J. Mutations: types and causes. Molecular Cell Biology. 2000;4.

81. Hijikata A, Tsuji T, Shionyu M, Shirai T. Decoding disease-causing mechanisms of missense mutations from supramolecular structures. Scientific reports. 2017;7(1):8541.

82. Hershberger RE, Norton N, Morales A, Li D, Siegfried JD, Gonzalez-Quintana J. Coding sequence rare variants identified in MYBPC3, MYH6, TPM1, TNNC1, and TNNI3 from 312 patients with familial or idiopathic dilated cardiomyopathy. Circulation: Cardiovascular Genetics. 2010;3(2):15561.

83. Dhandapany PS, Sadayappan S, Xue Y, Powell GT, Rani DS, Nallari P, et al. A common MYBPC3 (cardiac myosin binding protein $\mathrm{C}$ ) variant associated with cardiomyopathies in South Asia. Nature genetics. 2009;41(2):187-91.

84. Domínguez F, Cuenca S, Bilińska Z, Toro R, Villard E, Barriales-Villa R, et al. Dilated cardiomyopathy due to BLC2-associated athanogene 3 (BAG3) mutations. Journal of the American College of Cardiology. 2018;72(20):2471-81.

85. D Marshall J, Maffei P, B Collin G, K Naggert JJCg. Alstrom syndrome: genetics and clinical overview. 2011;12(3):225-35.

86. Arola AM, Sanchez X, Murphy RT, Hasle E, Li H, Elliott PM, et al. Mutations in PDLIM3 and MYOZ1 encoding myocyte $Z$ line proteins are infrequently found in idiopathic dilated cardiomyopathy. Molecular genetics and metabolism. 2007;90(4):435-40.

87. Schönberger J, Wang L, Shin JT, Do Kim S, Depreux FF, Zhu H, et al. Mutation in the transcriptional coactivator EYA4 causes dilated cardiomyopathy and sensorineural hearing loss. Nature genetics. 2005;37(4):418-22.

88. Carniel E, Taylor MR, Sinagra G, Di Lenarda A, Ku L, Fain PR, et al. a-Myosin heavy chain: a sarcomeric gene associated with dilated and hypertrophic phenotypes of cardiomyopathy. Circulation. 2005;112(1):54-9.

89. Zhang SB, Liu YX, Fan LL, Huang H, Li JJ, Jin JY, et al. A novel heterozygous variant p.(Trp538Arg) of SYNM is identified by whole-exome sequencing in a Chinese family with dilated cardiomyopathy. Annals of human genetics. 2019;83(2):95-9.

90. García-Pelagio KP, Chen L, Joca HC, Ward C, Lederer WJ, Bloch RJ. Absence of synemin in mice causes structural and functional abnormalities in heart. Journal of molecular and cellular cardiology. 2018;114:354-63.

91. Kirk EP, Sunde M, Costa MW, Rankin SA, Wolstein O, Castro ML, et al. Mutations in cardiac T-box factor gene TBX20 are associated with diverse cardiac pathologies, including defects of septation and valvulogenesis and cardiomyopathy. The American Journal of Human Genetics. 2007;81(2):280-91. 
92. Zhang J, Felder A, Liu Y, Guo LT, Lange S, Dalton ND, et al. Nesprin 1 is critical for nuclear positioning and anchorage. Human molecular genetics. 2009;19(2):329-41.

93. Ripoll-Vera T, Gámez JM, Govea N, Gómez Y, Núñez J, Socías L, et al. Clinical and prognostic profiles of cardiomyopathies caused by mutations in the troponin T gene. Revista Española de Cardiología. 2016;69(2):149-58.

94. Almomani R, Verhagen JM, Herkert JC, Brosens E, van Spaendonck-Zwarts KY, Asimaki A, et al. Biallelic truncating mutations in ALPK3 cause severe pediatric cardiomyopathy. Journal of the American College of Cardiology. 2016;67(5):515-25.

95. Valdés-Mas R, Gutiérrez-Fernández A, Gómez J, Coto E, Astudillo A, Puente DA, et al. Mutations in filamin $\mathrm{C}$ cause a new form of familial hypertrophic cardiomyopathy. Nature communications. 2014;5:5326.

96. Sveinbjornsson G, Olafsdottir EF, Thorolfsdottir RB, Davidsson OB, Helgadottir A, Jonasdottir A, et al. Variants in NKX2-5 and FLNC Cause Dilated Cardiomyopathy and Sudden Cardiac Death. Circulation: Genomic and Precision Medicine. 2018;11(8):e002151.

97. Reynolds PR, Mucenski ML, Le Cras TD, Nichols WC, Whitsett JA. Midkine is regulated by hypoxia and causes pulmonary vascular remodeling. Journal of Biological Chemistry.

2004;279(35):37124-32.

98. Wang Z-Y, Qu Q-Q, Seufert W, Taguchi T, Testa JR, Whitmore S, et al. Molecular cloning of the cDNA and chromosome localization of the gene for human ubiquitin-conjugating enzyme 9. Journal of Biological Chemistry. 1996;271(40):24811-6.

99. Johnson JO, Pioro EP, Boehringer A, Chia R, Feit H, Renton AE, et al. Mutations in the Matrin 3 gene cause familial amyotrophic lateral sclerosis. Nature neuroscience. 2014;17(5):664-6.

100. Müller TJ, Kraya T, Stoltenburg-Didinger G, Hanisch F, Kornhuber M, Stoevesandt D, et al. Phenotype of matrin-3-related distal myopathy in $16 \mathrm{G}$ erman patients. Annals of neurology. 2014;76(5):669-80. 